\title{
Spatial processing in human auditory cortex: The effects of 3D, ITD, and ILD stimulation techniques
}

\author{
Kalle J. Palomäki ${ }^{\mathrm{a}, \mathrm{b}, *}$, Hannu Tiitinen ${ }^{\mathrm{b}, \mathrm{c}}$, Ville Mäkinen ${ }^{\mathrm{b}, \mathrm{c}}$, Patrick J.C. May ${ }^{\mathrm{b}, \mathrm{c}}$, Paavo Alku ${ }^{\mathrm{a}}$ \\ ${ }^{\mathrm{a}}$ Laboratory of Acoustics and Audio Signal Processing, Helsinki University of Technology, P.O. Box 3000, \\ FIN-02015 HUT, Finland \\ b Apperception \& Cortical Dynamics (ACD), Department of Psychology, University of Helsinki, Finland \\ ${ }^{\mathrm{c}}$ BioMag Laboratory, Engineering Centre, Helsinki University Central Hospital, Helsinki, Finland
}

Accepted 10 February 2005

\begin{abstract}
Here, the perception of auditory spatial information as indexed by behavioral measures is linked to brain dynamics as reflected by the $\mathrm{N} 1 \mathrm{~m}$ response recorded with whole-head magnetoencephalography (MEG). Broadband noise stimuli with realistic spatial cues corresponding to eight direction angles in the horizontal plane were constructed via custom-made, individualized binaural recordings (BAR) and generic head-related transfer functions (HRTF). For comparison purposes, stimuli with impoverished acoustical cues were created via interaural time and level differences (ITDs and ILDs) and their combinations. MEG recordings in ten subjects revealed that the amplitude and the latency of the N1m exhibits directional tuning to sound location, with the amplitude of the right-hemispheric N1m being particularly sensitive to the amount of spatial cues in the stimuli. The BAR, HRTF, and combined ITD + ILD stimuli resulted both in a larger dynamic range and in a more systematic distribution of the $\mathrm{N} 1 \mathrm{~m}$ amplitude across stimulus angle than did the ITD or ILD stimuli alone. Further, the righthemispheric source loci of the N1m responses for the BAR and HRTF stimuli were anterior to those for the ITD and ILD stimuli. In behavioral tests, we measured the ability of the subjects to localize BAR and HRTF stimuli in terms of azimuthal error and front-back confusions. We found that behavioral performance correlated positively with the amplitude of the N1m. Thus, the activity taking place already in the auditory cortex predicts behavioral sound detection of spatial stimuli, and the amount of spatial cues embedded in the signal are reflected in the activity of this brain area.
\end{abstract}

(C) 2005 Elsevier B.V. All rights reserved.

Theme: Sensory systems

Topic: Auditory systems: central physiology

Keywords: Magnetoencephalography (MEG); N1m; Auditory; Spatial; Localization cues

\section{Introduction}

Localizing sound sources is particularly important for directing attention towards events in the auditory environment, and the detection of sound source direction is crucial for survival in many species. The human auditory system exploits sound localization cues embedded in auditory sig-

\footnotetext{
* Corresponding author. Laboratory of Acoustics and Audio Signal Processing, Helsinki University of Technology, P.O. Box 3000, FIN-02015 HUT, Finland. Fax: +358 9460224.

E-mail address: kalle.palomaki@hut.fi (K.J. Palomäki).
}

nals (for reviews, see [2,12]). The primary cues for azimuthal sound source localization are the interaural time and level differences (ITD and ILD, respectively), which are caused by a differential distance between the sound source and the ears and the acoustical shadowing effects of the head. Elevation is resolved through utilizing the spectral cues arising from the filtering effects of the pinna, the head, and the body. The physiological basis of spatial localization has been extensively studied using animal models (for reviews, see $[7,24]$ ) where sites sensitive to ITD, ILD, and spectral cues [39] and topographical mappings of auditory space $[7,22]$ have been found in the midbrain. In the primary auditory cortex, certain 
neurons have spatial receptive fields [3], and are organized in clusters of similar directional tuning [7].

With the introduction of non-invasive brain imaging techniques measuring the blood flow as well as the electric and magnetic activity of the human brain, research on the neural underpinnings of sound localization in humans has gained momentum. While functional magnetic resonance imaging (fMRI) and positron emission tomography (PET) provide for good accuracy in identifying activated brain areas, the temporal dynamics of sound source localization can be more precisely tackled with the study of rapid changes in neuronal currents. These are reflected in electroand magnetoencephalography (EEG and MEG, respectively) $[10,15]$ where cortical responses to sensory stimulation can be measured with an excellent temporal accuracy in terms of the event-related potential (ERP) and magnetic field (ERF). In the auditory domain, the $\mathrm{N} 1 \mathrm{~m}$ deflection and its derivative mismatch negativity (MMN) are commonly used to index activation of auditory cortex [19,26,27,31].

The MEG is sensitive to magnetic interference caused by loudspeakers and any other electro-acoustic transducer placed near the measurement device. Therefore, an acoustic tube sound system with compromised audio quality, delivering a two-channel mock description of the auditory environment, is typically used for transmitting auditory stimuli directly to the left and right ear of the subject. This, however, is already sufficient for studying the processing of ITD and ILD modulations in spatial stimuli. Importantly, MEG research $[11,32,33]$ has benefited from the recent development of headphone-based 3D-sound technology such as head-related transfer functions (HRTF), which provide for an accurate (re-)presentation of a realistic 3D sound field using binaural stimulus presentation through MEG-compatible earphones. Contrasting ITD and ILD modifications, whereby the subject experiences the sound as originating inside the head, 3D sounds are perceived to occur in specific locations outside the head. Thus, 3D sound technology provides for a marked improvement in the experienced three-dimensional aspect of stimulation which, consequently, widens the scope of investigations concerning the cortical processing of auditory space.

Previous MEG and EEG studies have revealed that activation in the auditory cortices of both hemispheres is stronger for contralateral auditory stimulation $[11,32$ $35,37,51,52]$. These findings are further corroborated by results obtained in animal models [14,18]. However, unlike in animal models [53], the dominant role of the righthemispheric auditory areas in sound localization in humans has been highlighted in measurements of the human brain $[17,21,32,33,54]$, and in studies on patients with righthemispheric lesions $[8,9,53]$. Previously, using non-individualized HRTF stimuli presented in the azimuthal plane, we found that the auditory $\mathrm{N} 1 \mathrm{~m}$ is more prominent for stimuli presented in the contralateral hemifield, and, importantly, that the amplitude of the $\mathrm{N} 1 \mathrm{~m}$ elicited by spatial stimuli exhibits almost twice as large a dynamic range in the right auditory cortex than in the left $[32,33]$. We also found that right-hemispheric preponderance holds across different types of stimulation, with speech stimuli increasing the N1m amplitude by nearly a factor of two compared to nonspeech stimuli [33]. However, differences in the hemispheric dominance across individuals has also been reported [11]. Interestingly, behavioral studies have found that, in binaural listening, subjects localize more accurately stimuli in the left hemifield [4], and in monaural listening conditions, subjects localize more accurately with their left ear [6], which indicates a right-hemispheric dominance in auditory localization.

The cortical processing of spatially impoverished (e.g., ITD and ILD) and binaural vs. spectral cues have been investigated in separate studies. Ungan et al. [46] found that ERP responses to ITD- and ILD-modulated stimuli had significantly different scalp topographies. Schröger [40] found that combined ITD/ILD deviants elicited a largeramplitude MMN than deviants containing ITD or ILD cues alone. Both of these EEG studies suggested that ITD and ILD cues are processed by different cortical mechanisms. Moreover, in MEG measurements exploiting individual HRTF-based stimulation only, Fujiki et al. [11] found that azimuthal (mainly binaural) cues are processed earlier than elevation (spectral) cues, which led the authors to suggest that auditory cortex may treat spectral cues differently from binaural ones. Related conclusions were made by Kaiser et al. [20], who suggested that the ITD cues were processed earlier (100-140 ms) than the spectral variation in the stimuli (around $180 \mathrm{~ms}$ ).

Hemodynamic measures $[5,13,25,48,54]$ appear to have found areas in the brain which are specifically activated by spatial sound stimulation. In general, on the basis of these findings, it seems that parietal brain areas have a special role in the processing of spatial content in auditory stimulation. For example, Bushara et al. [5], using PET, discovered areas specific to auditory (as opposed to visual) spatial localization in the superior parietal and prefrontal cortices indicating that auditory spatial processing extends well beyond the temporal auditory areas. Further, Zatorre et al. [54] found that spatial stimuli presented simultaneously from different locations elicit activity in posterior auditory cortex indicating that this area is involved in disambiguating overlapping auditory sources. Moreover, they found that inferior parietal cortex is specifically activated during a spatial localization task with the strength of this activation correlating with localization accuracy in terms of absolute error.

Based on the abovementioned data, several issues remain to be clarified. Firstly, there exist very little data on the possible differences between cortical activity elicited by spatial stimuli containing prominent spatial cues vs. impoverished spatial stimuli containing only ITD and ILD cues. In our understanding, no MEG study specifically comparing the effects of spatially enriched 3D and impoverished ITD and ILD stimuli on brain dynamics has 
been conducted. Secondly, the observations using 3D sound technology are almost exclusively based on non-individualized HRTF stimuli which fail to take into account individual differences in the pinna, the head, and the body. Thirdly, although it appears that the right auditory cortex processes spatial information with a higher resolution, a relationship between auditory cortical activity and behavioral performance remains unestablished. Fourthly, although activations of parietal brain areas have been found to be linked with the behavioral localization accuracy, the link between the temporal brain areas and behavioral localization remains unestablished. Consequently, the auditory-cortex activity picked up by EEG/MEG measures, likely to precede parietal brain activity, remains unlinked with the results obtained via hemodynamic measures.

Here, we aim to shed light on the above issues by firstly assessing how the human auditory brain areas process spatial information as indexed by the $\mathrm{N} 1 \mathrm{~m}$, the most prominent cortical response originating from the auditory cortex. We contrasted the processing of realistic, externally experienced 3D stimuli to that of spatially impoverished, internally experienced stimuli containing isolated ITD or ILD cues or their combinations. Importantly, for realistic stimuli we used not only generic HRTFs but also individualized spatial sounds constructed separately for each subject. Further, we carried out a behavioral test whereby the subjects performed a localization task of the 3D stimuli. Based on our previous observations $[32,33]$, we hypothesize that the strength of the activation in the auditory cortices reflects the spatial location of the auditory stimulus so that as the sound source is moved from an ipsi- to a contralateral location the auditory N1m increases in amplitude in a monotonic fashion. In order to test this hypothesis, we assess the amplitude organization of the $\mathrm{N} 1 \mathrm{~m}$ and correlate this measure to behavioral localization accuracy in order to establish a link between observable behavior and brain activity.

\section{Methods}

\subsection{Subjects}

Twelve volunteers (11 male, 1 female, age range 26-45) served as subjects with informed consent and with the approval of the Ethical Committee of Helsinki University Central Hospital. All the subjects reported normal hearing. The subjects participated in the MEG measurements and in the behavioral listening tests. The data of two subjects were discarded due to problems in measurement and determination of the $\mathrm{N} 1 \mathrm{~m}$ amplitude.

\subsection{Stimuli and sound delivery system}

The stimuli were delivered directly to the subject's ears with a custom-made acoustic tubephone sound system whose frequency response was digitally equalized in the $100 \mathrm{~Hz}-10 \mathrm{kHz}$ range (yielding maximal deviation $\pm 5 \mathrm{~dB}$; $\pm 2.5 \mathrm{~dB}$ in the $150-1500 \mathrm{~Hz}$ range). We constructed stimuli in five categories: two were realistic spatial 3D sounds presented from eight horizontal directions, and three were presented laterally via modifications of interaural (ITD and ILD) cues. The stimuli in each category were $50-\mathrm{ms}$ bursts of uniformly distributed noise (sampling rate $22050 \mathrm{~Hz}$ ) presented randomly from one of the eight locations with an (onset-to-onset) interstimulus interval of $750 \mathrm{~ms}$. Uniformly distributed noise was used in order to provide localization cues over a wide spectral range and to avoid the activation of speech-specific processes (see [33]).

The 3D sounds were presented from the horizontal plane from directions $0^{\circ}, 45^{\circ}, 90^{\circ}, 135^{\circ}, 180^{\circ},-135^{\circ},-90^{\circ}$, and $-45^{\circ}$ (see Fig. 1) through the earphones using either (a) individual BinAural Recordings (BAR) or (b) non-individualized (generic) HRTFs. The BAR stimuli were constructed individually for each subject: Recording microphones were attached to the entrance of the subject's ear canals, which were blocked using silicon paste. The 50-ms noise bursts (rectangular time window; no onset-offset ramps) were sequentially presented through eight loudspeakers placed around the subjects at a distance of $1.5 \mathrm{~m}$. The recorded signals contain a $50-\mathrm{ms}$ direct sound component which is accompanied and followed by room reflections which decay exponentially after stimulus offset $(60 \mathrm{~dB}$ within $300 \mathrm{~ms}$ ). The room used in the recordings was slightly reverberant (measured reverberation time $T_{60}=$ $0.3 \mathrm{~s}$ ) adhering to the IEC-268-13 standard [16] for listening rooms. The chain of playback and recording systems introduced a short, 1 - to 1.5 -ms onset ramp to stimuli.

The non-individualized [49], generic HRTF stimuli were produced by convolving a 50 -ms noise burst (1.4-ms onset/ offset ramps, hanning-windowing) with the head-related impulse responses (HRIR) of the abovementioned directions. The 1.4-ms onset ramp was selected to match that of

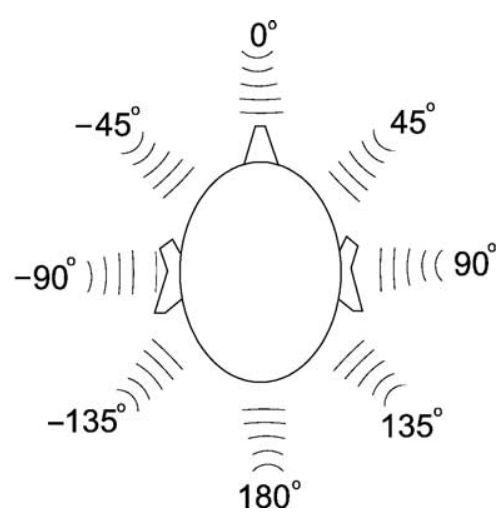

Fig. 1. Illustration of the stimulus presentation. Stimuli are presented spatially (perceived to occur in specific locations outside the head) to the subject through earphones from eight directions in the horizontal plane using realistic 3D sound stimulation (BAR and HRTF), or laterally (as originating inside the head) using the impoverished counterparts of the $3 \mathrm{D}$ stimuli (ITD + ILD, ITD, and ILD, see Section 2.2). 
the individualized BAR stimuli. The HRIRs used here were originally measured by Wightmann and Kistler [50]. From their set, we chose HRTFs for the subject SOU. As their measurement covered the horizontal plane in $10^{\circ}$ intervals, we constructed the HRIRs for the $45^{\circ}, 135^{\circ},-45^{\circ}$, and $-135^{\circ}$ directions by interpolating between the HRIRs of the two nearest azimuthal angles whereby the HRIRs were firstly transformed to a minimum phase form (MPF; see [23]) and, thereafter, averaged. The time delay was reconstructed by adding a delay line whose length was an average of the delay of two HRIRs used in the interpolation.

The laterally presented, internally experienced counterparts for the 3D stimuli were constructed via the use of ITD + ILD, ITD, and ILD modifications. In order to ensure their spectral correspondence with the 3D stimuli, 50-ms noise bursts (1.4-ms onset/offset ramps, hanning-windowing) were filtered to match the grand-averaged spectra of the BAR stimuli originating from the $0^{\circ}$ direction. The spectrum of the $0^{\circ}$ direction sample was selected because it represents (near) equal left- and right-ear spectra. The matching of the spectral envelopes was estimated by designing a low-order (30) finite impulse response filter (FIR) to model the spectra obtained from 512-point FFTs and grand-averaged across the subjects. A low-order model was used to remove spectral cues which might have made the stimuli appear externalized rather than internalized. A miscalculation was made in the filter design which caused the magnitude response to be slightly different from the desired one $(<1 \mathrm{~dB}$ up to $1 \mathrm{kHz},<5 \mathrm{~dB}$ up to $6 \mathrm{kHz},<10 \mathrm{~dB}$ up to $9 \mathrm{kHz}$ ). These variations, however, are only related to the spectral shape of the stimuli and have no effect on ITD and ILD cues embedded in the lateralized stimuli.

The construction of the lateralized stimuli involved two phases: firstly, an estimate of an ITD or an ILD corresponding to each horizontal angle was computed for each subject on the basis of the BAR stimuli and, secondly, these values were averaged over the subject population yielding a single non-individualized ITD and ILD value for each horizontal angle. For the ITD computation, the first $23 \mathrm{~ms}$ of the BAR stimulus were used in order to capture the direct sound component. The ITD values were computed using an auditory model where: (1) the left- and right-ear inputs were divided into frequency bands using a gamma-tone filter bank (center frequencies spaced uniformly between $200 \mathrm{~Hz}$ and $9900 \mathrm{~Hz}$ in the equivalent rectangular bandwidth scale) to simulate frequency resolution of peripheral auditory processing [36]; (2) the resulting signals were half-wave rectified to simulate auditory nerve firing patterns; (3) the corresponding left- and right-ear frequency bands were cross-correlated; (4) the normalized crosscorrelation functions of all the frequency bands were summed to form a pooled cross-correlation function from which the largest peak was selected to represent the ITD value of the sound sample (for a similar approach, see [41]). The numerical values of the ITDs were $0.00,0.39,0.70$, $0.41,0.00,-0.41,-0.70$, and $-0.39 \mathrm{~ms}$ for the azimuth angles $0^{\circ}, 45^{\circ}, 90^{\circ}, 135^{\circ}, 180^{\circ},-135^{\circ},-90^{\circ}$, and $-45^{\circ}$, respectively.

For the computation of the ILD values, the first $10 \mathrm{~ms}$ of the BAR stimuli were used, again, to prevent the reverberant sound field from affecting the estimate. The ILD values were computed as the difference in decibels between the left and right ear. The numerical values of the ILDs were -0.16 , $-11.67,-12.13,-7.07,-0.10,6.21,11.90$, and $11.07 \mathrm{~dB}$ for the azimuth angles $0^{\circ}, 45^{\circ}, 90^{\circ}, 135^{\circ}, 180^{\circ},-135^{\circ}$, $-90^{\circ}$, and $-45^{\circ}$, respectively. For simplicity, we will from now on index the ITDs and ILDs by the value of the corresponding azimuth angle.

The binaural loudness of the stimuli was balanced to 10 sones using a loudness model by Moore and Glasberg [30]. The gain of the audio system was calibrated to give $74 \mathrm{~dB}$ A-weighted Sound Pressure Level (SPL) measured for the $0^{\circ}$ stimulus at the earpiece of the tubephone system using a custom-made model of the ear canal and a TES 1351 type 2 SPL meter. This procedure ensured that approximately the same binaural loudness was used across the different experimental conditions and subjects.

\subsection{Behavioral task}

We carried out a behavioral test where the subject's task was to localize the 3D stimuli. The BAR and HRTF stimuli from the eight directions were presented in a random order, and the subject used a computer mouse to point the horizontal direction of the stimuli in a circle. If a subject was unsure about the stimulus direction angle, he/she was instructed to press a miss button. We used both the tubephone sound system utilized in the MEG measurement, and replicated the test using Sennheiser HD 580 headphones. This allowed a comparison between localization performance with the tubephone sound system and highquality commercial headphones $(16 \mathrm{~Hz}-30 \mathrm{kHz},-3 \mathrm{~dB})$. The order of the tasks and stimuli was counterbalanced across subjects. In each task, the subject performed 480 localizations.

Localization performance was measured in terms of the mean angular report and error, the number of front-back confusions, and the number of misses. Prior to analyses, front-back confusions, that is, a stimulus arriving from the front $\left(45^{\circ}, 0^{\circ},-45^{\circ}\right)$ perceived as arriving from behind $\left(135^{\circ}, 180^{\circ},-135^{\circ}\right)$ or vice versa, were automatically reversed in the subject's report of the direction angle (according to the autoreverse method introduced in [43] and now widely applied, e.g., $[4,50])$. For each subject, the mean angular reports were obtained as the average of the responses per stimulus direction. For statistical analyses, angular reports for back directions were represented by their equivalent frontal azimuths (i.e., in the same cone of confusion), and front-back confusions were analyzed separately. The mean angular error was obtained by first calculating the absolute value of the difference between the target and the reported direction and subsequently averag- 
ing the errors separately for each subject and stimulus direction.

\section{4. $M E G$ recordings}

Magnetic responses were recorded (passband 0.03-200 $\mathrm{Hz}$, sampling rate $600 \mathrm{~Hz}$ ) with a 306-channel whole-head magnetometer (Vectorview, Elekta Oy, Finland). The subject sat in a reclining chair under instruction not to pay attention to the auditory stimuli and to concentrate on watching a selfselected silent film. Over 100 instances of each stimulus type and direction angle were presented to each subject. The responses were averaged and baseline-corrected with respect to a 100-ms pre-stimulus period and bandpassfiltered at 2-30 Hz. Horizontal (HEOG) and vertical (VEOG) electrodes were used for automatically removing eye-movement related artifacts $(>150 \mu \mathrm{V})$.

The N1m peak amplitude and latency were quantified and analyzed from the gradiometer sensor pairs picking up maximal activity above the temporal lobes of the left and right hemisphere. The N1m source location was estimated using unrestricted equivalent current dipoles (ECDs) [15]. Separate subsets of 44 gradiometer sensors over the temporal areas of the left and right hemisphere were used in the ECD estimation. The head-based coordinate system was defined by the $x$-axis passing through the preauricular points (positive to the right), the $y$-axis passing through the nasion, and the $z$-axis as the vector cross product of the $x$ and $y$ unit vectors. ECDs were discarded $(15 \%$ and $1 \%$ of the data in the left and right hemispheres, respectively) from the analyses if their goodness-of-fit (GOF) was below $60 \%$ or if they were outliers. In order to quantify the noise characteristics of the left and right hemisphere, a Signal-to-Noise Ratio (SNR) was computed separately for each subject and cortical hemisphere. In calculating the SNR, signal energy was defined as the square of the $\mathrm{N} 1 \mathrm{~m}$ amplitude and noise energy was estimated as the squared sum of the signal in a $100-\mathrm{ms}$ pre-stimulus interval.

The location of the source of the $\mathrm{N} 1 \mathrm{~m}$ response was also estimated by using the technique of minimum current estimation (MCE) [47]. Unlike ECD source estimation, which is based on the point-source assumption [15], MCE can represent several local or distributed sources thereby providing a description of the distribution of cortical activation. ECD and MCE techniques are formally compared in [42]. For MCE analysis, the data were preprocessed by low-pass filtering at $30 \mathrm{~Hz}$ and detrended over a 0 - to 300-ms post-stimulus interval. A realistic head model (standard-bem, NeuroMag) was used in which the head was divided into a grid of 1425 points. From this, 147-point cylindrically shaped regions of interest (ROI) were used for the left and right temporal lobes. For each ROI, a center point of activation (CPA) was computed as the center of gravity of the MCE data averaged in a $20-\mathrm{ms}$ time window centered around the peak of the N1m response. CPA $x$-, $y$-, and $z$-coordinates were used in statistical analyses of the location.

We hypothesized that the amplitude of the $\mathrm{N} 1 \mathrm{~m}$ reflects sound localization by being an increasing monotonic function of azimuthal angle as this is varied from ipsilateral to contralateral. With the front and back direction pairs grouped (and thus the reversals ignored), this leads to a descending and ascending $\mathrm{N} 1 \mathrm{~m}$ amplitude order of $90^{\circ}, 45^{\circ} \%$ $135^{\circ}, 0^{\circ} / 180^{\circ},-45^{\circ} /-135^{\circ}$, and $-90^{\circ}$ for the left- and right-hemispheric responses, respectively. We performed an Angular Organization Test (AOT) which yields a rankingorder distance measure describing how well the $\mathrm{N} 1 \mathrm{~m}$ amplitudes were organized according to the hypothesis. In the test, performed separately for each subject and stimulus type, the N1m responses for the different angles are ordered according to response amplitude. If this order exactly corresponds to the hypothesis, the distance equals to zero. If the order differs from the hypothesis, the ordinal distances between the hypothesized and observed positions are summed. This procedure yields a ranking-order scale ranging from 0 to 8 , which reflects how well the amplitude of the $\mathrm{N} 1 \mathrm{~m}$ corresponds to the hypothesis.

\subsection{Statistical analyses}

For N1m and behavioral data analysis, we used repeated measures $M$-way ANalysis Of VAriance (ANOVA) and Newman and Keuls for post hoc comparisons. In order to test the hemispheric dominance of the N1m amplitude, we performed a two-way ANOVA where the hemisphere and direction angle were dependent factors. For the interaction analysis, the N1m amplitude data were organized to "pair" the medial, contra-, and ipsilateral responses between the left $\left(0^{\circ}, 45^{\circ}, 90^{\circ}, 135^{\circ}, 180^{\circ},-135^{\circ},-90^{\circ},-45^{\circ}\right)$ and right $\left(0^{\circ},-45^{\circ},-90^{\circ},-135^{\circ}, 180^{\circ}, 135^{\circ}, 90^{\circ}, 45^{\circ}\right)$ hemispheres.

In the AOT, we used non-parametric Friedman ANOVA for ranked order data and Wilcoxon tests for post hoc examination. We further tested how the results of the AOT, reflecting the organization of the left- and right-hemispheric $\mathrm{N} 1 \mathrm{~m}$ amplitude according to stimulus direction angle, correlate with behavioral responses as indexed by the average angular error for the BAR and HRTF stimuli.

\section{Results}

\subsection{Behavioral task}

Fig. 2 shows the grand-averaged results of reported direction angle (top panel) and angular error (bottom panel) for the BAR (left) and the HRTF (right) stimuli presented through the tubephone system. The stimulus direction had a statistically significant effect on the reported direction angle for both the BAR $(F[7,63]=474.24 ; P<0.001)$ and HRTF $(F[7,63]=378.87 ; P<0.001)$. Post hoc analyses revealed that the subjects were capable of discriminating stimulus 

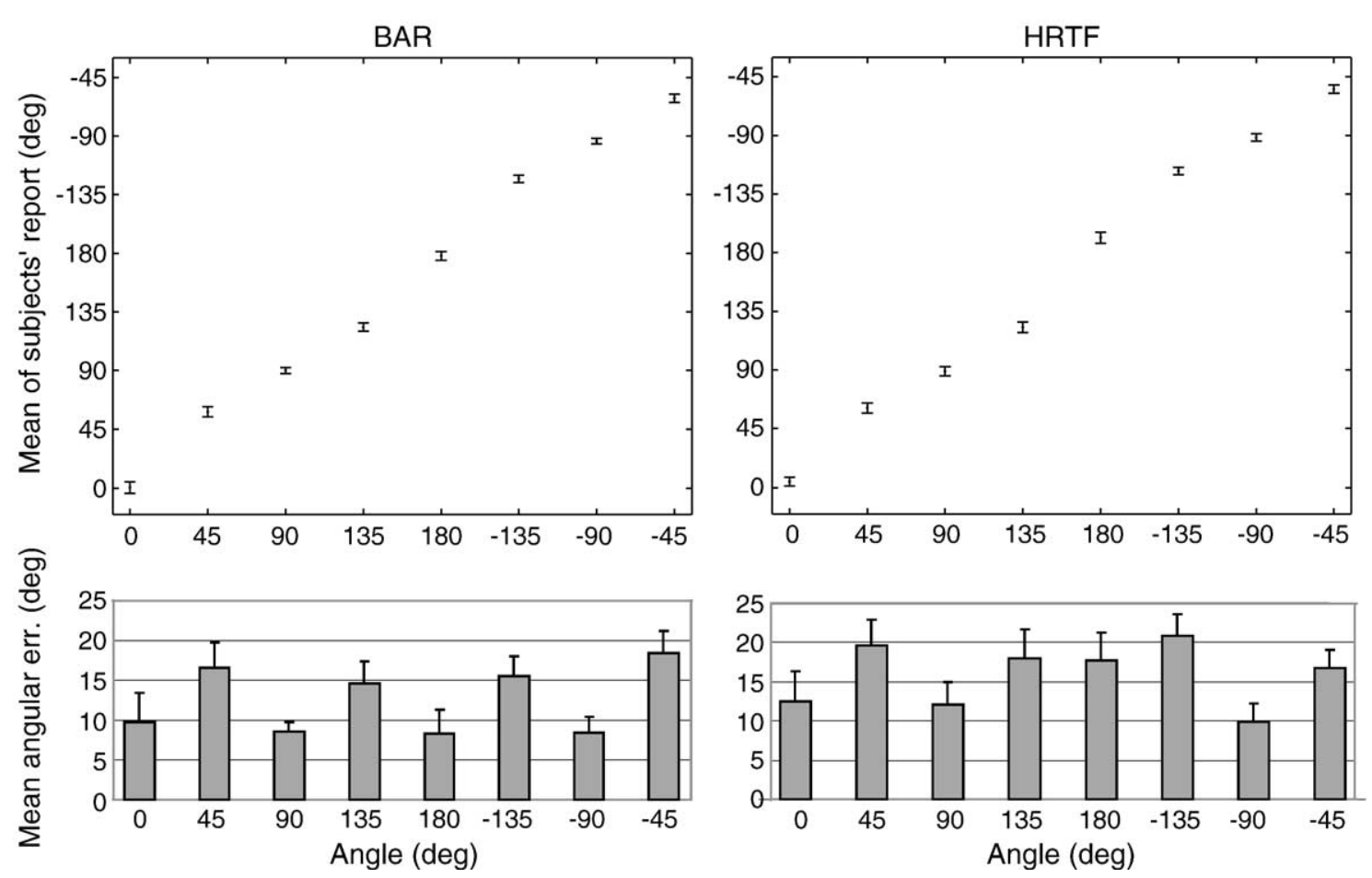

Fig. 2. Results on the localization task for the 3D stimuli (BAR and HRTF). Direction angle of subjects' behavioral report (grand-averaged over 10 subjects) for eight direction angles of the BAR (top-left) and the HRTF (top-right) stimuli. Front-back confusions in the subjects' reports were corrected. Angular error of the subjects' report (grand-averaged over 10 subjects) for the BAR (bottom-left) and HRTF (bottom-right) stimuli. Error bars indicate standard error of mean (SEM) which is calculated between the means of each subject's response for each direction. Near-linear relationship between the mean of subjects report and stimulus direction is observed for the BAR and HRTF stimuli. The angular errors are smaller for the (individualized) BAR than the (non-individualized) HRTF stimuli.

directions from one another (for the BAR stimuli, $P<0.001$ in all comparisons except the autoreversed front-back azimuth pairs, all of which were non-significant $P=$ n.s.; for the HRTF stimuli, $P<0.001$ in all comparisons, except between $0^{\circ}$ and $180^{\circ}$ where $P<0.01$, and $P=$ n.s. for all other autoreversed pairs).

In terms of the mean angular error, the BAR stimuli were localized more accurately than the HRTF stimuli (BAR $12.53^{\circ}$, HRTF $\left.15.94^{\circ} ; F[1,9]=8.78 ; P<0.05\right)$. The amount of front-back confusions were slightly smaller for BAR than for $\operatorname{HRTF}(24.5 \%$ and $32.2 \%$, respectively; $F[1,9]=$ $4.20 ; P=0.07)$. Further, the mean angular error varied according to stimulus direction for both the $\operatorname{BAR}(F[7,63]=$ 3.92; $P<0.01)$ and $\operatorname{HRTF}(F[7,63]=2.69 ; P<0.05)$ stimuli. On the average, the angular error for the BAR stimuli presented on the front-back $\left(9.0^{\circ}\right)$ and left-right $\left(8.5^{\circ}\right)$ axes was smaller than that for the stimuli presented from oblique angles $\left(16.3^{\circ}\right)$. The number of misses for the BAR and HRTF stimuli were $1.2 \%$ and $1.8 \%$, respectively.

The BAR stimuli were localized slightly more accurately when delivered through headphones than tubes (angular error using tubes $12.5^{\circ}$ vs. headphones $9.7^{\circ} ; F[1,9]=10.53$; $P<0.05$; front-back confusions using tubes $24.5 \%$ vs. headphones $18.9 \% ; F[1,9]=5.02 ; P=0.052)$. The HRTF stimuli were also localized more accurately with headphones than tubes in terms of front-back confusions (tubes
$32.2 \%$, headphones $27.1 \%, F[1,9]=5.24 ; P<0.05)$, whereas the differences in the angular error (tubes $15.9^{\circ}$, headphones $\left.14.7^{\circ}\right)$ were not statistically significant $(P=$ n.s.). The number of misses using the headphones were $0.6 \%$ and $2.0 \%$ for the BAR and HRTF stimuli, respectively. Although the deviations in the frequency responses of the tubephone system were rather large, the above comparisons indicate relatively small differences between localization accuracy when using tubephones vs. headphones. This implies that the static deviations in the frequency response of the tubephone system are associated with the original sound source and have only a minor effect on spatial localization.

\subsection{The N1m amplitude}

Both the spatially enriched 3D (BAR, HRTF) and impoverished (ITD + ILD, ITD, ILD) stimuli elicited prominent $\mathrm{N} 1 \mathrm{~m}$ responses in both the left and right hemisphere, with the near-dipolar field patterns indicating sources in the vicinity of the auditory cortex of each hemisphere (Fig. 3). Analyses of the amplitude of the N1m (Fig. 4) revealed that in both the left and right hemisphere, the direction angle had significant main effects $(P<0.01$ in all analyses; Table 1). In both cortical hemispheres, the N1m responses elicited by stimuli from the contralateral hemifield 


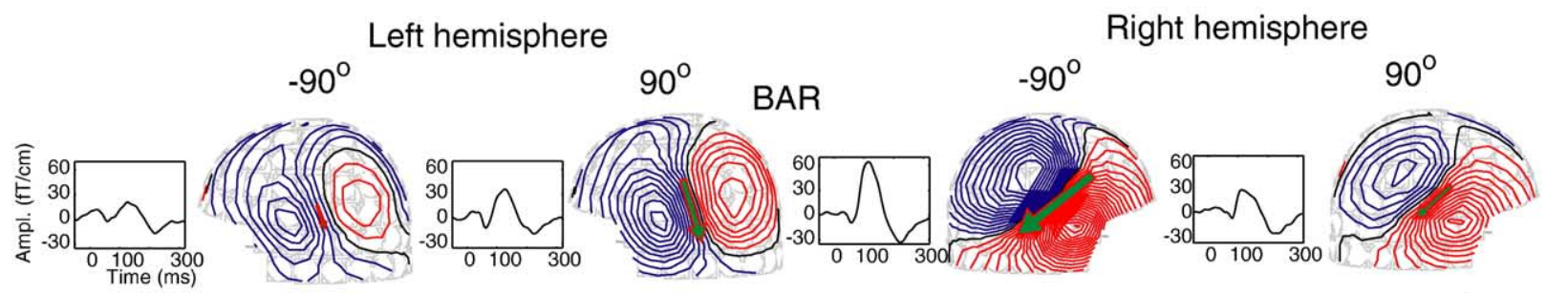

$t=125.5 \mathrm{~ms}, Q=4.7 \mathrm{nAm} \quad t=121.8 \mathrm{~ms}, Q=12.5 \mathrm{nAm}$

$t=112.7 \mathrm{~ms}, Q=18.8 \mathrm{nAm}$

$t=116.3 \mathrm{~ms}, Q=9.3 \mathrm{nAm}$

HRTF
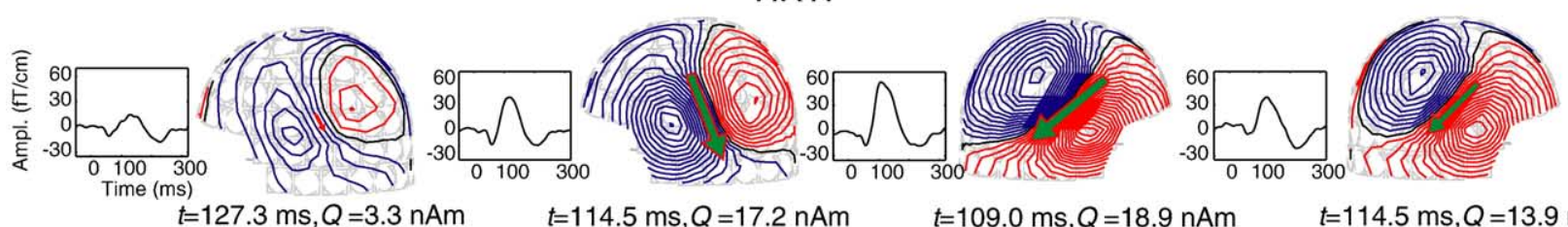

$t=127.3 \mathrm{~ms}, Q=3.3 \mathrm{nAm}$

$t=114.5 \mathrm{~ms}, Q=17.2 \mathrm{nAm}$

$t=109.0 \mathrm{~ms}, Q=18.9 \mathrm{nAm}$

$t=114.5 \mathrm{~ms}, Q=13.9 \mathrm{nAm}$

ITD+ILD
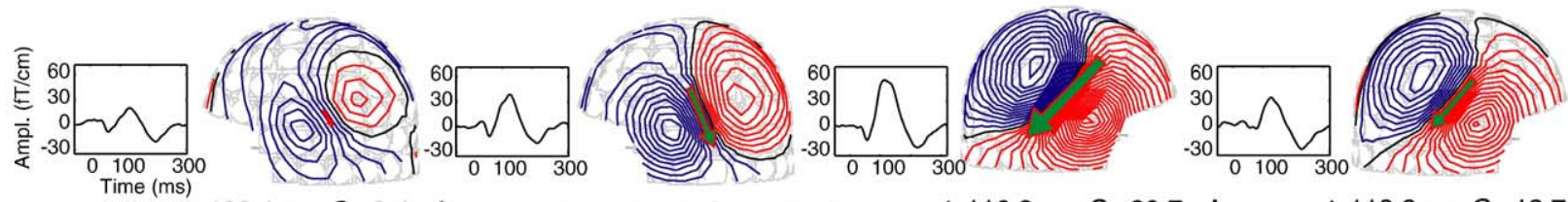

$t=129.1 \mathrm{~ms}, Q=3.4 \mathrm{nAm}$

$t=123.7 \mathrm{~ms}, Q=12.2 \mathrm{nAm}$

$t=116.3 \mathrm{~ms}, Q=20.7 \mathrm{nAm}$

$t=118.2 \mathrm{~ms}, Q=12.7 \mathrm{nAm}$

ITD
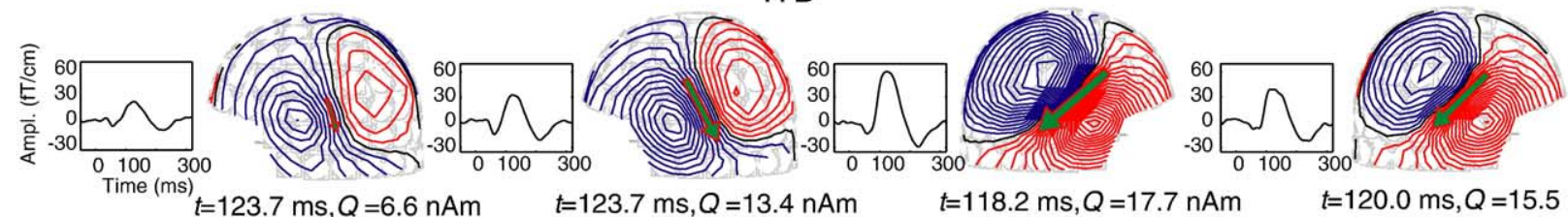

$t=123.7 \mathrm{~ms}, Q=6.6 \mathrm{nAm}$

$t=123.7 \mathrm{~ms}, Q=13.4 \mathrm{nAm}$

$t=118.2 \mathrm{~ms}, Q=17.7 \mathrm{nAm}$

$t=120.0 \mathrm{~ms}, Q=15.5 \mathrm{nAm}$

ILD
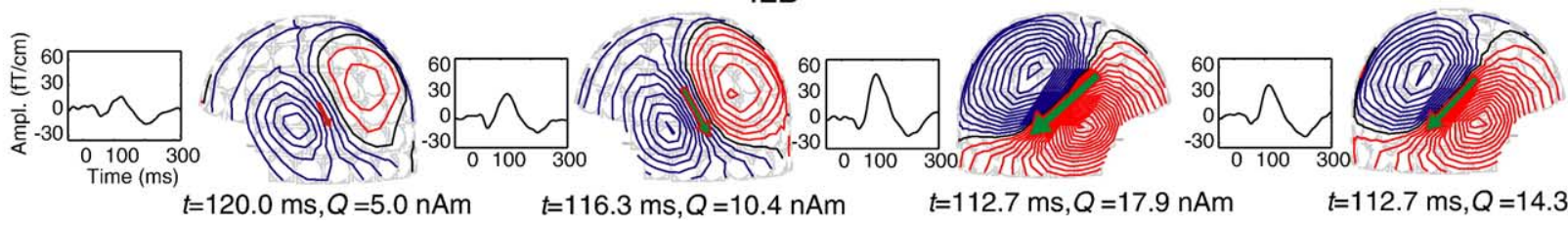

$116.3 \mathrm{~ms}, Q=10.4 \mathrm{nAm}$

$t=112.7 \mathrm{~ms}, Q=17.9 \mathrm{nAm}$

$t=112.7 \mathrm{~ms}, Q=14.3 \mathrm{nAm}$

Fig. 3. Illustration on the evoked responses and equivalent current dipole source location (ECD) for the N1m in the left and right hemisphere. The figure shows evoked responses (grand-averaged over 10 subjects) from the gradiometer channel picking up the maximal N1m amplitude, and ECDs (contour step $10 \mathrm{fT} / \mathrm{cm}$ ) for direction angles $-90^{\circ}$ and $90^{\circ}$ of the BAR, HRTF, ITD + ILD, ITD, and ILD stimuli. More prominent contralateral activation over the left and right temporal lobes is observed. Overall, the responses in the right hemisphere are larger than in the left.

were larger than those elicited by stimuli originating in the ipsilateral hemifield. That is, stimuli originating from the left hemifield elicited larger right-hemispheric responses than the stimuli from the right hemifield, and vice versa. The N1m amplitudes were organized less consistently for the ipsilateral than contralateral stimuli.

The responses averaged over stimulus type and direction angle were larger in the right than left hemisphere $(F[1,9]=$ $7.84, P<0.05)$. This effect was consistent across subjects, as only one subject out of ten had slightly larger $(7 \mathrm{fT} / \mathrm{cm})$ responses in the left hemisphere. However, the interaction term (Factors: Hemisphere $\times$ Stimulus type $\times$ Direction angle; $F[28,252]=2.26, P<0.001)$ indicated that the $\mathrm{N} 1 \mathrm{~m}$ amplitude varied across stimulus type and direction angle. In separate analyses per stimulus type, the amplitudes of the $\mathrm{N} 1 \mathrm{~m}$ in the right hemisphere were found to be, on average, larger than those in the left hemisphere for the BAR (47 fT/ $\mathrm{cm}$ and $79 \mathrm{fT} / \mathrm{cm}$ in the left and right hemisphere, respectively; $F[1,9]=4.35, P=0.067)$, HRTF $(52 \mathrm{fT} / \mathrm{cm}$ and $90 \mathrm{fT} / \mathrm{cm} ; F[1,9]=8.90, P<0.05)$, ITD + ILD (42 fT/ $\mathrm{cm}$ and $77 \mathrm{fT} / \mathrm{cm} ; F[1,9]=12.21, P<0.01)$, ITD $(44 \mathrm{fT} / \mathrm{cm}$ and $81 \mathrm{fT} / \mathrm{cm} ; F[1,9]=5.58, P<0.05)$, and ILD $(40 \mathrm{fT} / \mathrm{cm}$ and $73 \mathrm{fT} / \mathrm{cm} ; F[1,9]=11.45, P<0.01)$ stimuli. The interaction term between the factors hemisphere and direction angle was statistically significant for the BAR stimuli $(F[7,63]=2.89, P<0.05)$, showing that $\mathrm{N} 1 \mathrm{~m}$ amplitude dynamics were larger in the right hemisphere in terms of contralateral maximum $\left(-90^{\circ}\right)$ and ipsilateral minimum $\left(90^{\circ}\right)$. That is, for the BAR stimuli, the righthemispheric $\mathrm{N} 1 \mathrm{~m}$ for contralateral stimuli was larger than the left-hemispheric N1m for contra- and ipsilateral stimuli $(P<0.001$ for both), and the right-hemispheric N1m for ipsilateral stimuli was larger than the left-hemispheric N1m for ipsilateral stimuli $(P<0.05)$. 

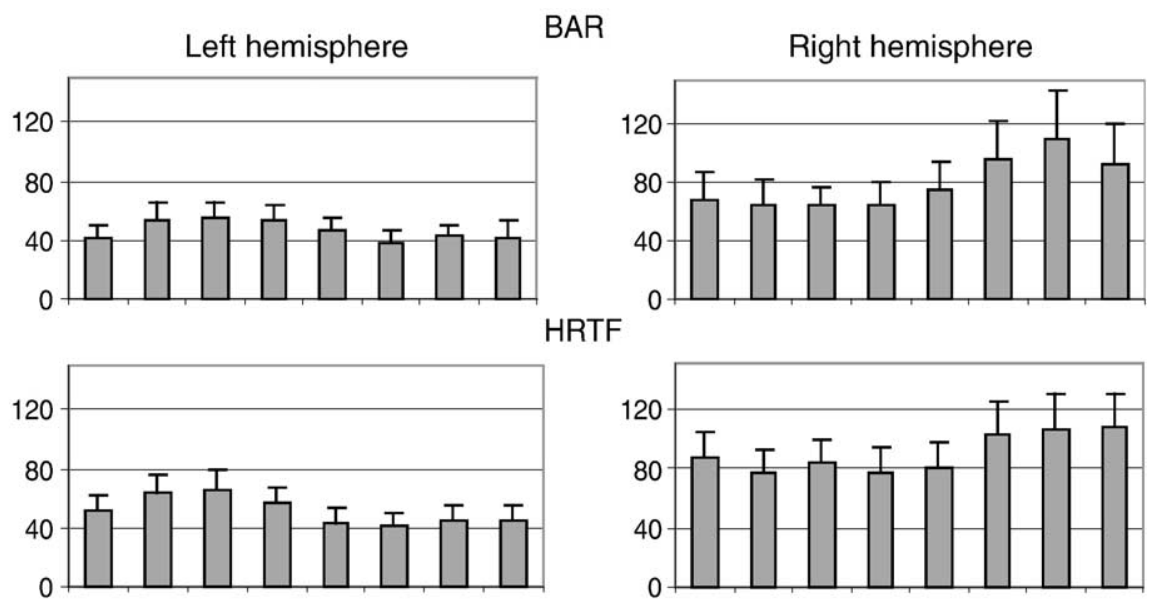

ITD+ILD
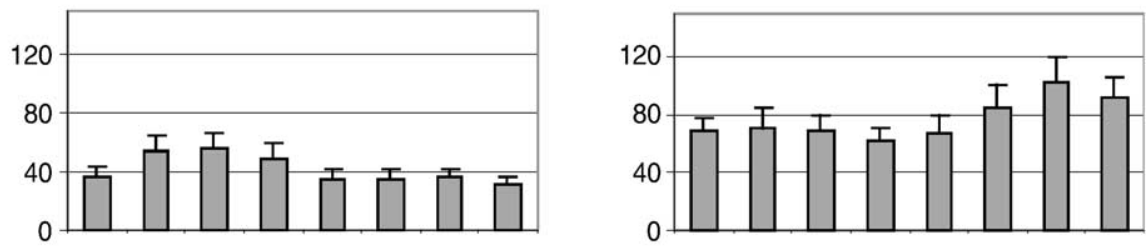

ITD
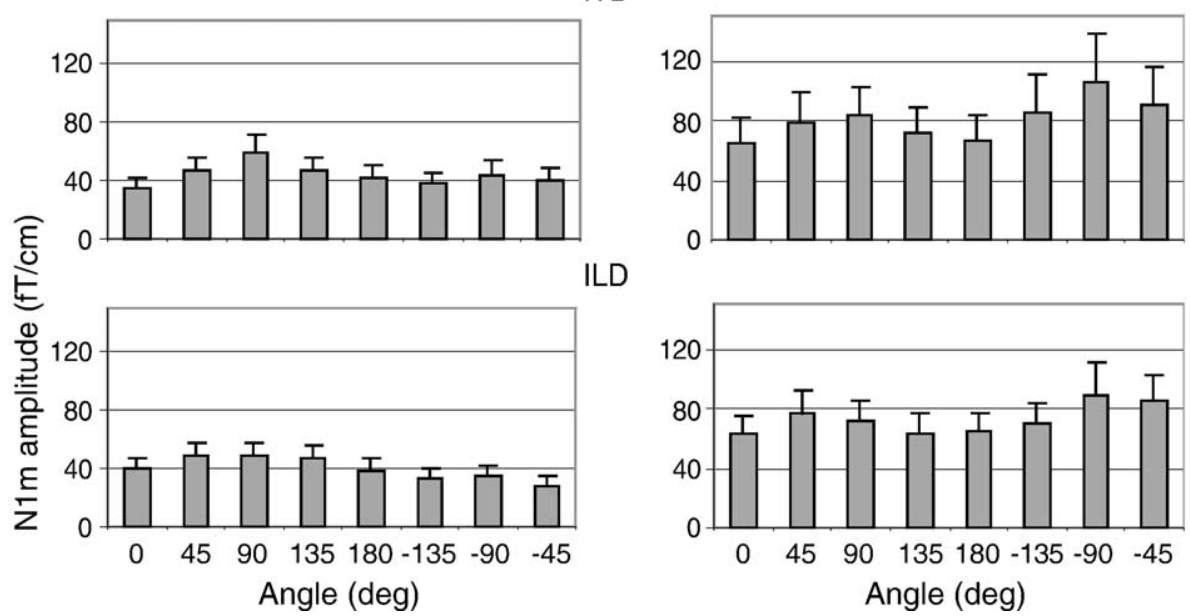

Fig. 4. The N1m amplitude (grand-averaged over 10 subjects) as a function of direction angle for the BAR, HRTF, ITD + ILD, ITD, and ILD stimuli. Error bars indicate SEM. N1m amplitude exhibits contralaterally prominent response patterns in both the left and right hemispheres. Responses are larger in the right hemisphere. Ipsilateral minima were observed in all conditions (except for the ITD stimuli in the right hemisphere, for which the minimum occurred for stimuli presented from $0^{\circ}$ angle).

While no such interaction effects were observed for any of the other stimulus types, the ILD stimuli, however, provided an exception to this rule in exhibiting a significant interaction between the factors hemisphere and direction

Table 1

ANOVA table for N1m amplitude as a function of stimulus direction for BAR, HRTF, ITD + ILD, ITD, and ILD stimuli

\begin{tabular}{lll}
\hline & Left hemisphere & Right hemisphere \\
\hline BAR & $F[7,63]=4.21, P<0.001$ & $F[7,63]=5.73, P<0.001$ \\
HRTF & $F[7,63]=9.51, P<0.001$ & $F[7,63]=6.44, P<0.001$ \\
ITD + ILD & $F[7,63]=13.38, P<0.001$ & $F[7,63]=11.11, P<0.001$ \\
ITD & $F[7,63]=8.49, P<0.001$ & $F[7,63]=4.31, P<0.001$ \\
ILD & $F[7,63]=9.40, P<0.001$ & $F[7,63]=3.65, P<0.01$ \\
\hline
\end{tabular}

angle $(F[7,63]=2.52, P<0.05)$. Rather than being a straightforward result of increased dynamics of the $\mathrm{N} 1 \mathrm{~m}$ amplitude (as observed for the BAR stimuli), this appears to be due to the right hemisphere exhibiting a double-peaked organization of the $\mathrm{N} 1 \mathrm{~m}$ amplitude across stimulus angle as opposed to the single-peaked one in the left hemisphere (see Fig. 4).

Stimulus type had a significant effect on the N1m amplitude only in the left hemisphere $(F[4,36]=3.14, P<$ 0.05). Post hoc analyses revealed that the amplitudes evoked by the HRTF stimuli were significantly larger than those evoked by the ITD + ILD $(P<0.05)$ and ILD $(P<$ $0.05)$ stimuli. However, caution is warranted in the interpretation of these data because the $\mathrm{N} 1 \mathrm{~m}$ in the left 
hemisphere $(\mathrm{SNR}=17 \mathrm{~dB})$ were noisier than in the right hemisphere $(\mathrm{SNR}=23 \mathrm{~dB} ; F[1,9]=129.63, P<0.001)$.

For the right-hemispheric $\mathrm{N} 1 \mathrm{~m}$, the interaction between stimulus type and the direction angle was significant $(F[28,252]=2.97, P<0.001)$, showing that the angular organization of the $\mathrm{N} 1 \mathrm{~m}$ amplitude varied across stimulus types. Notably, there were considerable differences in the dynamic range of the right-hemispheric N1m amplitude between left- and right-hemifield stimulation, with the amplitude of the N1m elicited by the BAR and ILD stimuli spanning the widest and narrowest range, respectively.

These observations lead us to develop more detailed analyses for the dynamics and angular organization of the $\mathrm{N} 1 \mathrm{~m}$ amplitude. For the analysis of the dynamics within each hemisphere, the $\mathrm{N} 1 \mathrm{~m}$ amplitudes for contralateral hemifield stimuli were subtracted from their ipsilateral counter parts (Fig. 5). This means that in the left hemisphere, the N1m amplitudes evoked by the left-hemifield $\left(-45^{\circ},-90^{\circ},-135^{\circ}\right)$ stimuli were subtracted from their right-hemifield $\left(45^{\circ}, 90^{\circ}, 135^{\circ}\right)$ counterparts. Similarly, in the right hemisphere, the $\mathrm{N} 1 \mathrm{~m}$ amplitudes for the righthemifield were subtracted from the left-hemifield stimuli. Stimulus type had a statistically significant effect in the dynamics in both the left $(F[4,36]=2.74, P<0.05)$ and right hemisphere $(F[4,36]=4.29, P<0.01)$. In the right hemisphere, the BAR stimulation spanned the largest dynamic range, and there were significant differences in response dynamics between the BAR and the ILD $(P<$ $0.01)$ and ITD $(P<0.05)$ stimulation. The dynamic ranges of the right-hemispheric $\mathrm{N} 1 \mathrm{~m}$ amplitude for the different stimulus types were, in descending order, BAR (35 fT/cm), HRTF (26 fT/cm), ITD + ILD (26 fT/cm), ITD $(16 \mathrm{fT} / \mathrm{cm})$, and ILD $(11 \mathrm{fT} / \mathrm{cm})$. In the left hemisphere, the ITD + ILD (19 fT/cm) stimulation spanned the largest dynamic range and ITD $(11 \mathrm{fT} / \mathrm{cm})$ the smallest, this effect not quite reaching statistical significance $(P=0.54)$.

The N1m amplitude organization in the left and the right hemisphere was analyzed using an angular organization test (AOT) which measures how smoothly the $\mathrm{N} 1 \mathrm{~m}$ amplitude increases as stimulus direction angle is rotated from the ipsito the contralateral direction. In both the left and right hemisphere, stimulus type had a significant effect on the result of the AOT (Fig. 5, $\chi^{2}[10,4]=10.49$ and $19.19 ; P<$ 0.05 and $P<0.001$ for the left and right hemisphere, respectively). In the right hemisphere, post hoc analyses revealed significantly better AOT results for the BAR (2.90), HRTF (2.90), and ITD + ILD (2.90) stimuli than for the ITD (4.60) and ILD (4.60) stimuli $(P<0.05)$. For the observations in the right hemisphere, we further tested how the results of the AOT correlate with individual behavioral responses (the mean angular error) for the BAR and HRTF stimuli. The correlation coefficients between the
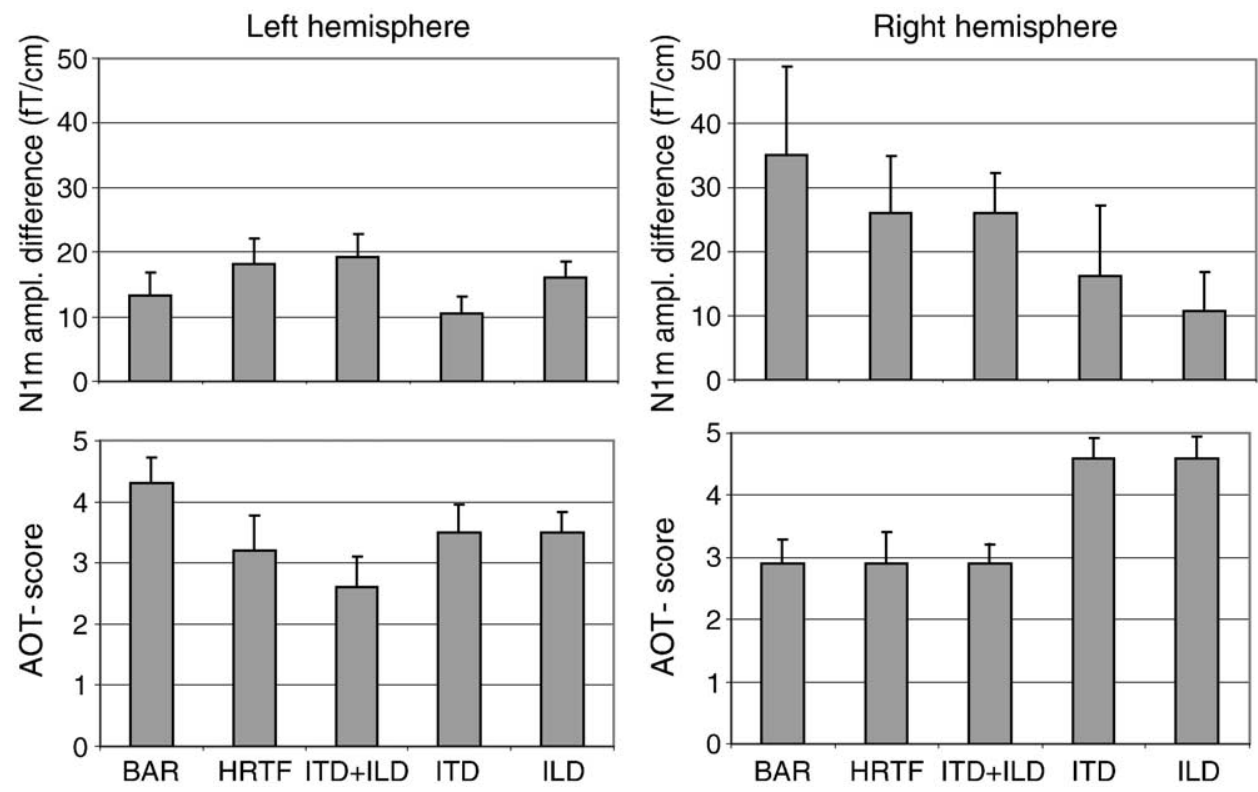

Fig. 5. The effects of N1m amplitude response dynamics (top) and angular organization (bottom). For the analysis of the dynamics (grand-averaged over 10 subjects) within each hemisphere, the $\mathrm{N} 1 \mathrm{~m}$ amplitudes for contralateral hemifield stimuli were subtracted from their ipsilateral counterparts. This means that in the left hemisphere, the N1m amplitudes evoked by the left-hemifield $\left(-45^{\circ},-90^{\circ},-135^{\circ}\right)$ stimuli were subtracted from their right-hemifield $\left(45^{\circ}, 90^{\circ}, 135^{\circ}\right)$ counterparts. Similarly, in the right hemisphere, the N1m amplitudes for the right-hemifield were subtracted from the left-hemifield stimuli. The response dynamics in the right hemisphere reflected the amount of spatial cues in the stimuli. The dynamic ranges of the right-hemispheric N1m amplitude for the different stimulus types were, in descending order, BAR, HRTF, ITD + ILD, ITD, and ILD. In the left hemisphere, the dynamical range was somewhat smaller, and the order of stimuli in terms of dynamics did not reflect the amount of spatial information. The angular organization test (AOT) for N1m amplitudes (grandaveraged over 10 subjects) in the right hemisphere shows that the amplitude patterns are scored significantly better for the stimuli containing simultaneously both ITD and ILD cues (BAR, HRTF, and ITD + ILD) comparing to spatially most impoverished stimuli with only ITD or ILD cues. 
mean angular error and the results of the AOT were $r^{2}=$ $0.80(P<0.01)$ and $r^{2}=0.51(P=$ n.s. $)$ for the BAR and HRTF stimuli, respectively. In the left hemisphere, exhibiting less systematic amplitude behavior, the AOT score for the HRTF (3.20) and ITD + ILD (2.60) stimuli was significantly smaller than that for the BAR stimuli (4.30; $P<0.05)$. Moreover, in the left hemisphere, the correlations between the mean angular error and AOT results were nonsignificant $\left(r^{2}=0.17\right.$ and -0.081 for BAR and HRTF, respectively; $P=$ n.s.). Taken together, these results indicate that behavioral localization ability of the subjects is reflected in the amplitude of the $\mathrm{N} 1 \mathrm{~m}$ according the hypothesized pattern, and that this link between behavior and brain dynamics is specific to the activity observed in the right hemisphere.

\subsection{The N1m latency}

The N1m occurred, on the average, at a latency of 132 and $125 \mathrm{~ms}$ in the left and right hemisphere, respectively, while the differences between them were not significant $(P=$ n.s.). When the effect of latency was tested separately for each stimulus type, it was found that N1m for ITD in the right hemisphere occurred significantly earlier than in the left $(F[1,9]=5.66, P<0.05)$. Tests on the other stimulus types did not yield statistically significant effects $(P=$ n.s. $)$. In the right hemisphere, stimulus direction angle affected the latency of the N1m elicited by the BAR (range: 119-131 $\mathrm{ms} ; F[7,63]=2.19, P<0.05)$, HRTF $(121-130 \mathrm{~ms}$; $F[7,63]=2.61, P<0.05)$, and ITD + ILD $(117-129 \mathrm{~ms}$; $F[7,63]=5.01, P<0.001$ ) stimuli (Fig. 6). No effects on the

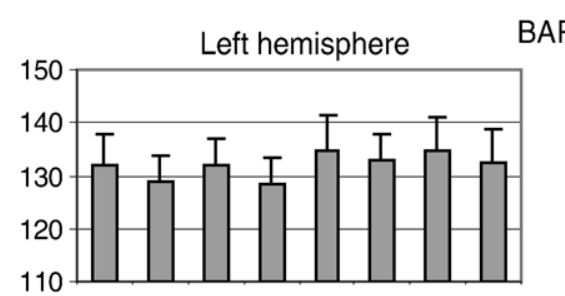

BAR

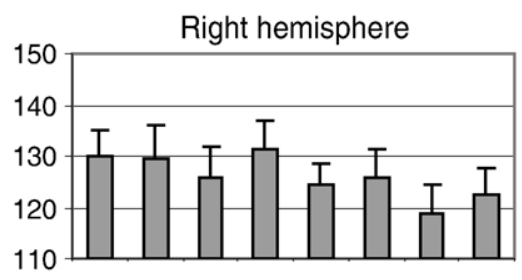

HRTF
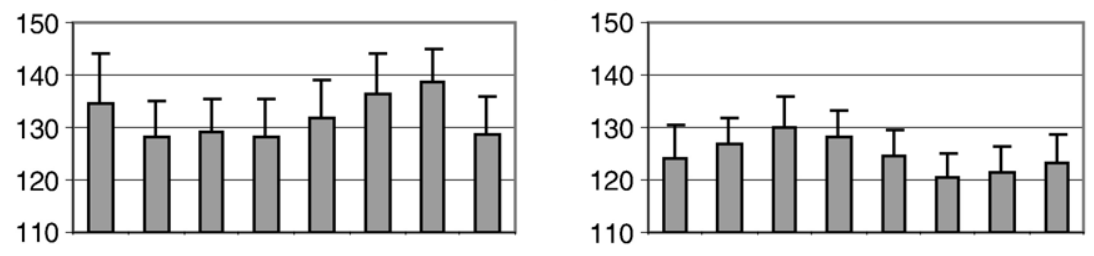

ITD+ILD
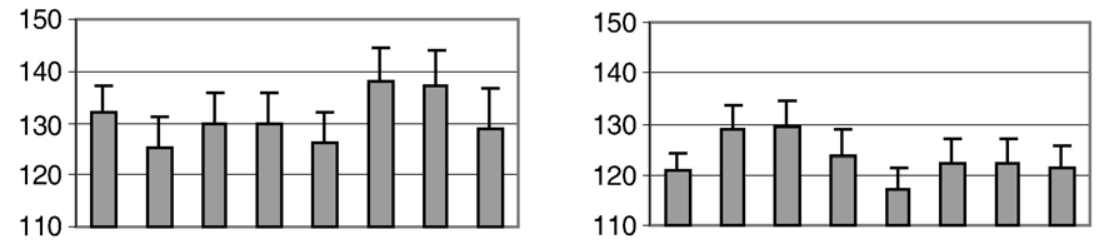

ITD
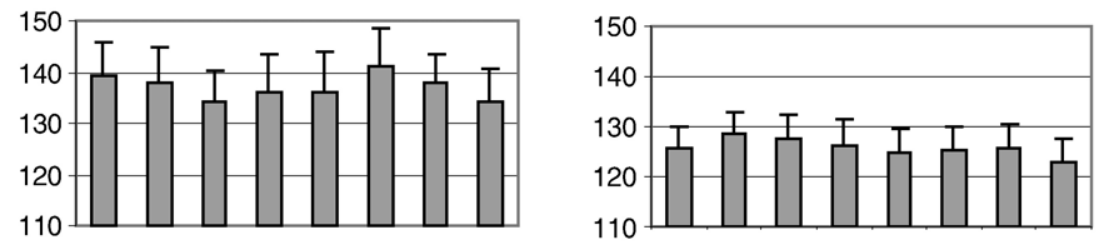

ILD
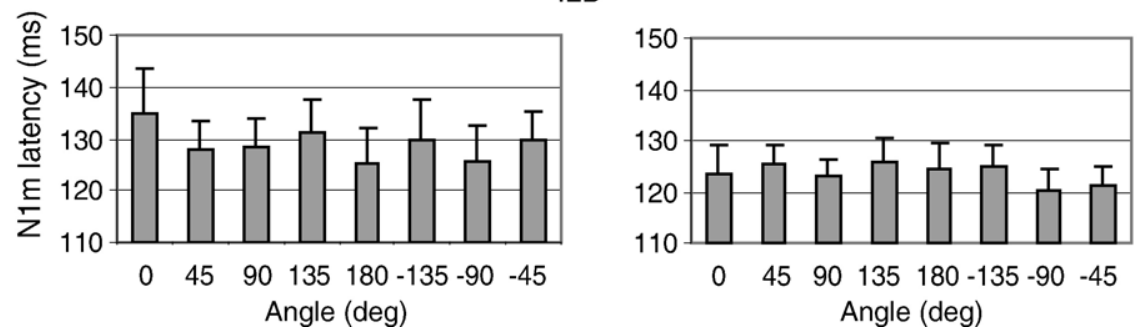

Fig. 6. The N1m latency (grand-averaged over 10 subjects) as a function of direction angle in the left and right hemispheres for the BAR, HRTF, ITD + ILD, ITD, and ILD stimuli. In the right hemisphere, significant variation in latency was found for stimuli containing simultaneous ITD and ILD cues (BAR, HRTF and ITD + ILD). Generally, latencies were shorter for contralateral than ipsilateral stimulation. 
latency of the N1m were observed for ITD and ILD stimulation $(P=$ n.s. $)$. For the BAR, HRTF, and ITD + ILD stimuli, the right-hemispheric N1m response latencies for left-hemifield stimulation were on average $6 \mathrm{~ms}$ shorter than for right-hemifield stimulation. In the left hemisphere, stimulus direction angle did not have statistically significant effects on the N1m latency ( $P=$ n.s.). On the basis of these results, the right hemisphere exhibits sensitivity to stimulus direction angle, expressed as latency shifts in the N1m, for enriched spatial stimuli (BAR and HRTF) and ITD + ILD, which all contain minimally both ITD and ILD cues.

There were no significant differences in the latency of the right-hemispheric $\mathrm{N} 1 \mathrm{~m}$ between the five stimulus types $(P=$ n.s.) whereas the latency of the left-hemispheric N1m varied across stimulus type $(F[4,36]=2.90, P<0.05)$. The latency of the N1m for the BAR and ILD stimuli was significantly shorter than that for ITD stimuli $(P<0.05$ in both comparisons).

\subsection{The N1m source location analysis}

We studied how stimulus type affected the source location of the N1m by analyzing possible changes in the $x-, y-$, and $z$-coordinates of the ECDs. In the right hemisphere, stimulus type had a statistically significant main effect on the (anterior-posterior) $y$-coordinate $(F[4,28]=3.26, P<0.05)$. Fig. 7 shows that the ECDs for the 3D stimuli (BAR and HRTF) were, on the average, $2.0 \mathrm{~mm}$ anterior to those of the ITD + ILD, ITD, and ILD stimuli. Post hoc analyses revealed that this effect was due

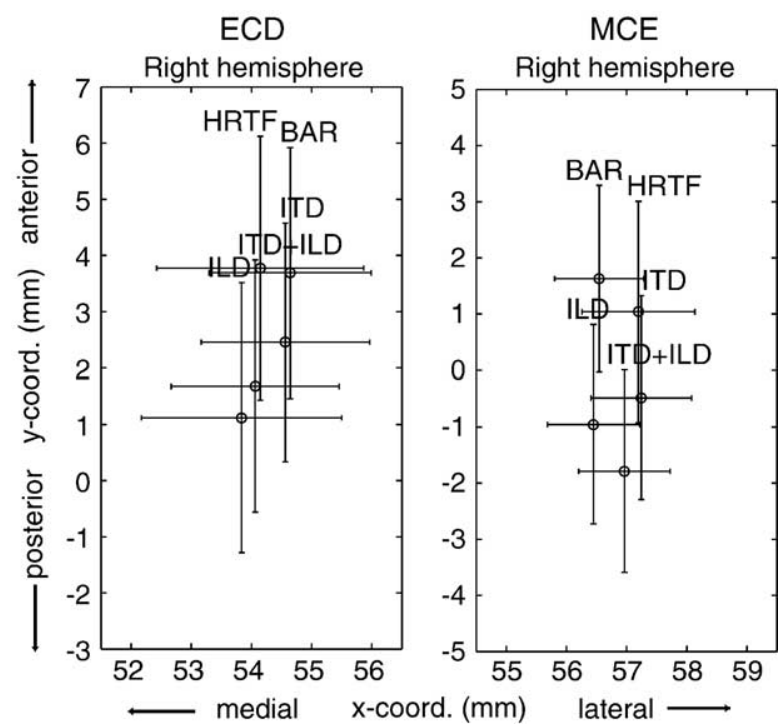

Fig. 7. Location coordinates $x$ (lateral medial) and $y$ (anterior-posterior) averaged over stimulus directions as obtained using equivalent current dipoles (ECD) (grand-averaged over 8 subjects) and a center point of activation for minimum current estimation (CPA-MCE) (grand-averaged over 10 subjects). Location coordinates for externally perceived realistic 3D stimuli (BAR and HRTF) are anterior compared to the laterally perceived spatially impoverished stimuli (ITD + ILD, ITD, and ILD). to the ECDs for the BAR stimuli being more anterior than those for the ILD stimuli $(P<0.05)$.

The effects of direction angle on the coordinates $x, y, z$ were also investigated separately for each hemisphere and stimulus type using ECDs of the N1m. In the right hemisphere, stimulus direction angle had a significant effect on the $y$-coordinate for the ITD + ILD stimuli (range: -0.2 to $3.5 \mathrm{~mm} ; F[7,63]=2.20, P<0.05)$, with the ECDs for the left-hemifield $\left(-45^{\circ},-90^{\circ},-135^{\circ}\right)$ stimuli being anterior to those for the right-hemifield $\left(45^{\circ}, 90^{\circ}, 135^{\circ}\right)$ and medial $\left(0^{\circ}\right.$, $180^{\circ}$ ) stimuli. However, post hoc analyses did not reveal significant differences for the direction angles. Similarly, for the ITD stimuli $(F[7,63]=3.71, P<0.01)$, post hoc analyses revealed that the ECDs for the $0^{\circ}$ angle $(-1.0 \mathrm{~mm})$ were posterior to those of the $90^{\circ}(4.1 \mathrm{~mm}),-90^{\circ}(5.2 \mathrm{~mm})$, and $-45^{\circ}(3.8 \mathrm{~mm})$ direction angles $(P<0.05$ for all comparisons). In the left hemisphere, direction angle had a significant effect only on the ECD $x$-coordinate for the HRTF stimuli $(F[7,35]=3.77, P<0.01)$. Post hoc analyses revealed that the ECDs for the $-135^{\circ}$ direction angle $(-57.5 \mathrm{~mm})$ were lateral to those of the $90^{\circ}(-52.2 \mathrm{~mm})$, $135^{\circ}(-52.4 \mathrm{~mm}), 180^{\circ}(-52.0 \mathrm{~mm})$, and $-45^{\circ}$ $(-55.1 \mathrm{~mm})$ angles $(P<0.05$ for all comparisons $)$.

We further analyzed the $\mathrm{N} 1 \mathrm{~m}$ source locations using minimum current estimates (MCEs; Fig. 8). Firstly, the effects of direction angle on the MCE coordinates in terms of the center point of activation (CPA) were investigated separately for each hemisphere and stimulus type. No significant effects, however, were found $(P=$ n.s. $)$. Secondly, we investigated the effects of the five stimulus types separately for each hemisphere. As in the ECD source estimation, we found that stimulus type had an effect on the $y$-coordinate of the CPA in the right hemisphere $(F[4,36]=$ 8.45, $P<0.001$; see Fig. 7 for a comparison of the two results). Post hoc analyses of the MCE data revealed that the CPAs for the BAR and HRTF stimuli were significantly more anterior (on the average, $2.4 \mathrm{~mm}$ ) than those for the ITD + ILD, ITD, and ILD stimuli $(P<0.05$ for all comparisons). Thus, it appears that 3D stimuli are processed in a different cortical location than stimuli containing ITD and ILD cues alone, or in combination.

\section{Discussion}

We studied how human auditory cortex processes auditory spatial information by utilizing MEG measurements and auditory stimuli ranging from realistic 3D sounds (individually constructed BARs and generic HRTFs) to spatially impoverished ones (containing ITDs and ILDs). Further, we related these cortical processes to auditory perception on a behavioral level. We found that the amplitude of the N1m was larger in the right hemisphere, where the dynamic range of the N1m across stimulus direction angle was larger for stimuli containing prominent spatial cues than for the spatially impoverished stimuli. This indicates that the right 


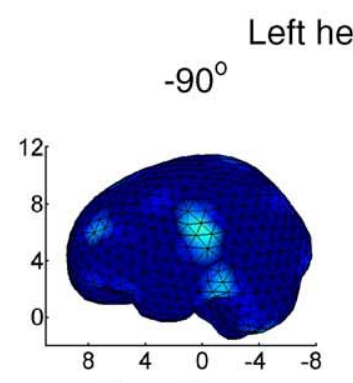

$t=115.5-135.5 \mathrm{~ms}$

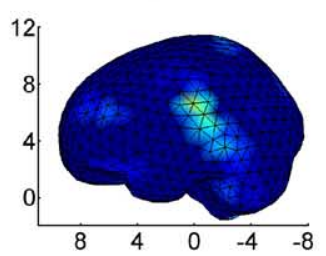

$t=117.3-137.3 \mathrm{~ms}$

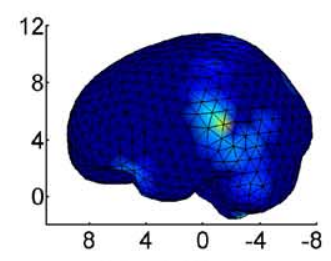

$t=119.1-139.1 \mathrm{~ms}$
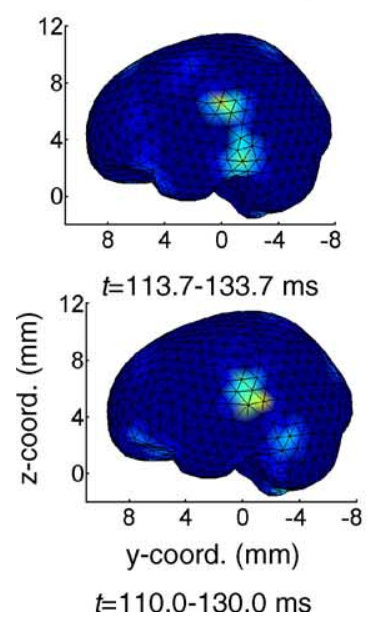

$90^{\circ}$

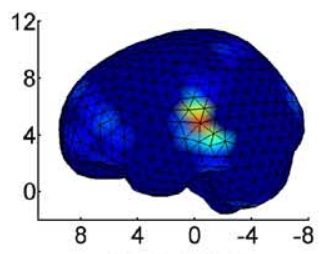

$t=111.8-131.8 \mathrm{~ms}$

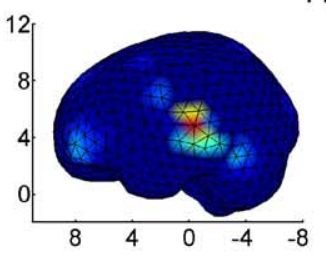

$t=104.5-124.5 \mathrm{~ms}$

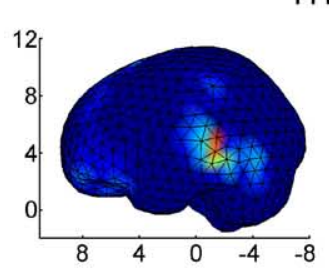

$t=113.7-133.7 \mathrm{~ms}$
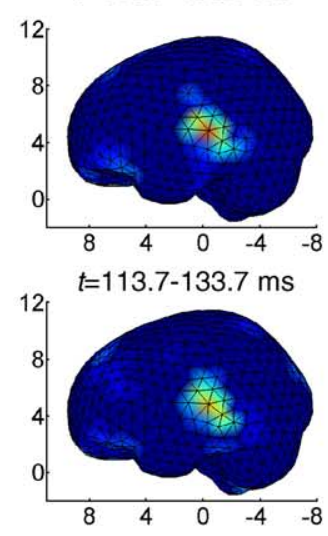

$t=106.3-126.3 \mathrm{~ms}$
BAR

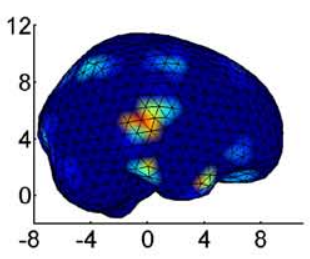

HRTF

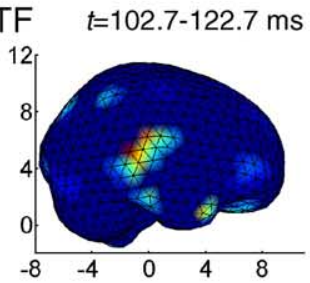

ITD+ILD

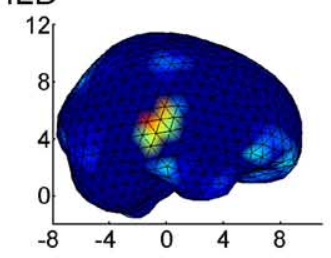

ITD
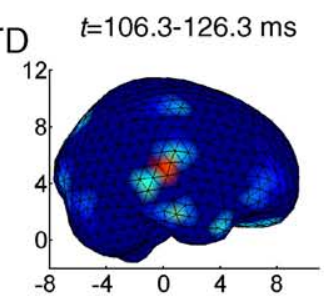

ILD $_{12} t=108.2-128.2 \mathrm{~ms}$

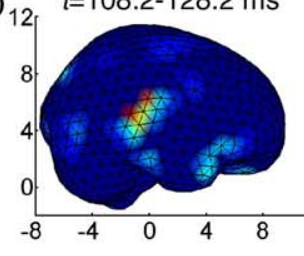

$t=102.7-122.7 \mathrm{~ms}$ $90^{\circ}$

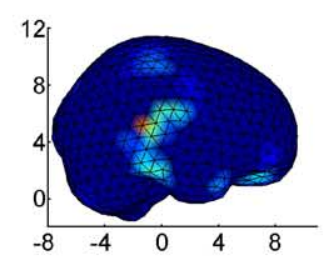

$t=106.3-126.3 \mathrm{~ms}$

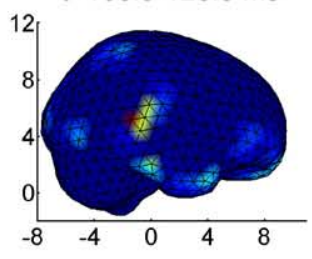

$t=104.5-124.5 \mathrm{~ms}$

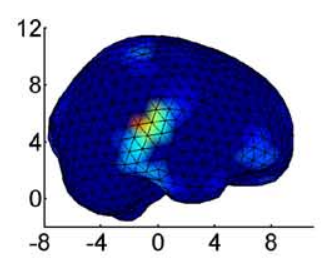

$t=108.2-128.2 \mathrm{~ms}$

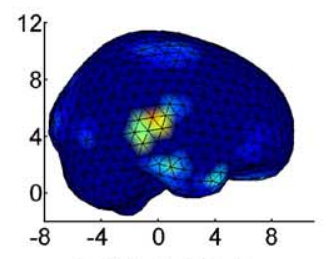

$t=110.0-130.0 \mathrm{~ms}$

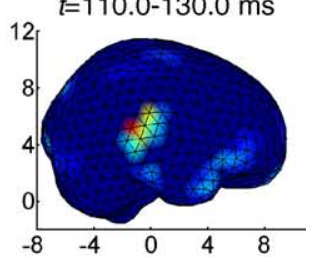

$t=102.7-122.7 \mathrm{~ms}$

Fig. 8. MCE activation for the N1m amplitude (grand-averaged over 10 subjects) over the left and right hemispheres, for direction angles $-90^{\circ}$ and $90^{\circ}$, and for the BAR, HRTF, ITD + ILD, ITD, and ILD stimuli. Contralaterally more prominent activation over the left and right temporal lobes was observed. Overall, the responses in the right hemisphere were larger than in the left.

auditory cortex has a dominant role in processing of spatial cues, and that the spatial information embedded in the stimulus further determines response dynamics. In general, amplitude dynamics appear to be roughly an additive function of the strength of the localization cues. Secondly, for both the 3D and combined ITD + ILD sounds, we found that the latency of the right-hemispheric N1m exhibits directional tuning to sound location. As no such effects were observed for ITD and ILD cues presented alone, it therefore appears that the combination of the two cues is a minimum requirement for this right-hemispheric latency coding of spatial location to emerge. Finally, using both ECDs and
MCEs, we found that the 3D stimuli elicited slightly more anteriorly displaced activity when compared to activity elicited by ITD and ILD modulations. Thus, the differences in the N1m amplitude and latency dynamics between 3D and spatially impoverished stimuli are, depending on stimulus material used, accompanied by differences in activity distribution in cortex.

Importantly, we assessed how brain dynamics are reflected in behaviorally measured spatial discrimination and found further evidence for the specialization of the right auditory cortex in the processing of spatial cues in auditory stimuli. The AOT revealed that the N1m of the right 
hemisphere reflected spatial cues more systematically than the left-hemispheric N1m: The right-hemispheric AOT index for the BAR, HRTF, and ITD + ILD stimuli was 2.90 whereas that for ITD and ILD alone was 4.60. We further validated these observations by measuring the correlation between the AOT results and the mean angular error of the behavioral results. This revealed that the organization of the righthemispheric $\mathrm{N} 1 \mathrm{~m}$ responses as indexed by AOT predicted behavioral performance, and the correlation turned out to be remarkably higher for the individually constructed BAR stimuli than for the generic HRTF stimuli. This is likely to result from the more realistic description of auditory spatial environment provided with the individualized stimuli. Overall, the above results indicate that the activity in the auditory cortex of the right hemisphere reflects how well spatial stimuli are discriminated perceptually and that a larger dynamic range of the $\mathrm{N} 1 \mathrm{~m}$ amplitude is likely to provide for better behavioral discrimination.

There is fairly good agreement that both hemispheres respond more vigorously to contralaterally presented auditory stimuli as shown by studies using monaural $[34,35,37,51,52]$ and spatial stimulation using $3 \mathrm{D}$ audio $[11,32,33]$. However, for stimuli presented laterally via ITD, some studies have reported contralaterally larger responses [28], while others have found no significant differences between contra- and ipsilateral stimulation [51]. These inconsistencies may be due to differences in stimulation methods (click train [28] vs. frequency sweep [51]). On the basis of the current results, however, it appears that for all types of spatial stimuli, including ITDmodulated stimuli, auditory cortices in both hemispheres respond contralaterally more prominently.

Corroborating the results of previous research $[4,6,17,21,32,33,53]$, we found that the right hemisphere of the human brain appears to be more dominant in spatial processing as indexed by the $\mathrm{N} 1 \mathrm{~m}$ amplitude. For the HRTF, ITD + ILD, ITD, and ILD stimuli, the N1m amplitude in the right hemisphere was larger than that in the left hemisphere. The dynamic range of the righthemispheric N1m elicited by the BAR stimuli was larger than for any other stimulus type used in this study. Thus, as large differences between responses to different angles seem to provide for better behavioral discrimination, the dynamic range of the $\mathrm{N} 1 \mathrm{~m}$ amplitude, as opposed to response amplitude per se, might be a better indicator for the hemispheric dominance specific to the processing of spatial content in stimulation.

The analyses of the right-hemispheric N1m amplitude patterns indicated a significant interaction between stimulus type and direction angle. This could be due to differences in the organization of the $\mathrm{N} 1 \mathrm{~m}$ amplitude according to stimulus direction angle and response dynamics for different stimulus types. When arranged in a descending order according to the magnitude of the dynamic range of the $\mathrm{N} 1 \mathrm{~m}$ amplitude, the stimulus types were BAR, HRTF, ITD + ILD, ITD, and ILD. The BAR stimuli contain natural ITDs,
ILDs, and spectral cues specific to the individual subject whereas the generic HRTFs contain ITD, ILD, and spectral cues which, however, do not replicate the unique properties of each individual listener. The amplitude range of the $\mathrm{N} 1 \mathrm{~m}$ for the combined ITD and ILD stimuli was found to reside between that of the BAR and HRTF stimuli and ITD- or ILD-alone stimuli. The latter two are weaker cues for sound location than when combined, or when appearing in combination with spectral cues present in BAR and HRTF stimuli. Taken together, these findings indicate that the amount of localization cues in the stimuli as well as their experienced quality (individualized/generic; external/internal) is reflected in the amplitude of the right-hemispheric $\mathrm{N} 1 \mathrm{~m}$ response.

Moreover, we found that the latency of the righthemispheric $\mathrm{N} 1 \mathrm{~m}$ exhibits directional tuning to sound location for BAR, HRTF, and ITD + ILD stimulation. For the ITD and ILD stimuli, no significant variation in response latency was found. This indicates that simultaneous presence of ITD and ILD cues is a minimum requirement for inducing latency variations in auditory cortical dynamics. In contrast, McEvoy et al. [28] found statistically significant latency shifts of the N1m over the right hemisphere as a function of ITD for click train stimuli. In the present study, the latency span of the $\mathrm{N} 1 \mathrm{~m}$ responses (12.3 $\mathrm{ms}$ for the BAR stimuli) was much larger than the ITD variation embedded in the stimuli (from -0.7 to 0.7 $\mathrm{ms}$, yielding a total difference of $1.4 \mathrm{~ms}$ ). Thus, the delay observed in the latency of the auditory N1m is a product of processing both the ITD and ILD cues, and is almost ten times larger than the actual delay in the stimuli. While we are left with no proper explanation for this phenomenon, our results are in line with the study by McEvoy et al. [28] who found latency shifts up to $11 \mathrm{~ms}$ corresponding to ITDs ranging from -0.7 to $0.7 \mathrm{~ms}$. No evidence that the $\mathrm{N} 1 \mathrm{~m}$ response latency is organized according to stimulus direction was evident in the left hemisphere, although the ITD stimulation resulted in longer response latencies than for other stimulus types. This observation may be due to a relative insensitivity of the left auditory cortex to spatial stimulus location, although it might also be a spurious noise-induced effect as indicated by the low SNR estimates.

Short and long latencies are usually associated with large and small N1m amplitudes, respectively [31], and our data agree with this general principle. The activation of auditory cortex in a spatial sound environment is due to several factors: the time and level differences, the differential transmission delays to cortex from the ipsi and contralateral ears, and the constellation of auditory cortex cells receiving different combinations of ascending excitatory and inhibitory connections from the contra- and ipsilateral ears [29]. Thus, depending on the sound source direction, net excitation in the auditory cortex could either precede or follow inhibition, thereby leading to faster, amplitudeenhanced or delayed, smaller-amplitude N1m responses, respectively [27]. 
Studies in animal models [24] have revealed that the ITD and ILD cues are differentially processed already in the early stages of the auditory pathway. Evidently, in order to produce a coherent spatial perception, these cues are integrated in later stages. This integration process is not well understood in other species than barn owls [44] regarded as ultimate masters of sound localization. Ungan et al. [46] and Schröger [40] studied whether ITD and ILD are processed differentially in terms of ERPs in humans. Schröger [40] applied an oddball paradigm, presenting frequently occurring standard stimuli in the midline (zero ILD and ITD) and infrequently occurring deviant stimuli laterally by modulating the ILD or the ITD alone or in combination. It was found that the ITD and ILD in combination elicited a MMN seemingly matching in amplitude the sum of the amplitudes of the MMN responses elicited by the stimuli containing ITD or ILD cues alone, thus indicating an additive effect. A related effect is evident in our results for the combined ITD + ILD stimuli and for the ITD and ILD stimuli alone. When summed, the dynamic ranges of N1m amplitude for the ITD $(16 \mathrm{fT} / \mathrm{cm})$ and ILD $(11 \mathrm{fT} / \mathrm{cm})$ stimuli were only slightly larger than the range for the ITD + ILD $(26 \mathrm{fT} / \mathrm{cm})$ stimuli. This indicates that the ITD and ILD may be combined with a near-linear process. Further, Ungan et al. [46] compared the processing of ITD and ILD cues in terms of the electrically measured N1 response. They found that ITD and ILD stimulation resulted in different scalp topographies and, further, that the dipole source location estimates for the responses elicited by these stimulus types had different loci and orientations. However, in the current study, we found no evidence for ITD and ILD stimulation resulting in different $\mathrm{N} 1 \mathrm{~m}$ amplitude patterns or source locations.

Considering the processing of spectral cues, two MEG studies on the MMN response have suggested that spectral cues are processed later than binaural ones $[11,20]$. In the present study, investigating the $\mathrm{N} 1 \mathrm{~m}$, we found that the loci of processing of 3D stimuli (BAR, HRTF) incorporating both binaural and spectral cues were anterior to the loci for the ITD and ILD stimuli containing only binaural cues. Thus, a shift in location of cortical activation might be related to the processing of the spectral cues which, in contrast to $[11,20]$, occur already at the latency span of the $\mathrm{N} 1 \mathrm{~m}$. With the aid of structural MRI, previous studies have found the $\mathrm{N} 1 \mathrm{~m}$ to be generated in the vicinity of auditory cortex $[19,38]$. The N1m is probably due to a complicated distribution of neural activity in auditory cortex, evolving both in time and space, for which the ECD model provides only an estimate of the "center of gravity". Recent results indicate that during the early, ascending part of the N1m (at about $85 \mathrm{~ms}$ ), posterior areas of auditory cortex are activated, and during the late, descending part of the $\mathrm{N} 1 \mathrm{~m}$ (at about $150 \mathrm{~ms}$ ), activity has shifted some $7 \mathrm{~mm}$ to anterior auditory areas [19]. The earlier portion of the N1m possibly encompasses Heschl's gyrus, planum temporale, the superior temporal sulcus, the middle temporal gyrus and posterior aspects of the superior temporal gyrus whereas the later portion of the $\mathrm{N} 1 \mathrm{~m}$ is generated anterior to primary auditory cortex. Thus, our results obtained at the peak latency of the $\mathrm{N} 1 \mathrm{~m}$ tentatively indicate that the time and intensity cues of spatial stimuli might be predominantly processed in Heschl's gyrus and accompanying areas whereas spectral cues activate areas anterior to primary auditory cortex.

In the behavioral experiment, the localization of the BAR stimuli was less accurate for the oblique angle directions than for front-back and left-right axes. This could be due to a response bias where subjects tend to localize sound sources at the left $\left(-45^{\circ},-135^{\circ}\right)$ and right $\left(45^{\circ}, 135^{\circ}\right)$ oblique angles as biased towards the left or right sides, respectively (for similar results, see [1], page 73). Studies on sound localization using virtual, full spherical presentation have typically reported small localization errors in the azimuth $[49,50]$ but large errors in the elevation originating from problems in localization within the cone of confusion regions [2] (e.g., front-back, up-down, etc., confusions). In our study, it is possible that the stimuli presented from left and right oblique angles were occasionally perceived to originate from high or low locations on the corresponding cone of confusion, and when required to indicate sound source location on the horizontal plane, the subjects projected those towards the left and right sides, respectively.

The importance of parietal brain areas in spatial localization has been highlighted in recent PET studies $[5,48,54]$. Specifically, it has been found that localization accuracy measured in terms of angular error correlates positively with the strength of parietal-lobe activation, indicating that listeners with least accurate localization made greatest demands on the parietal system [54]. In the present MEG study, we observed no parietal activity, but found a positive correlation between behavioral localization performance and the activation of the auditory cortex of the right hemisphere. Notably, the MEG measurements were conducted in the passive recording condition only (for active condition see e.g., [45]), and the behavioral localization performance was assessed in a separate session. Our results should therefore be seen as complementing the findings of [54], where recordings were carried out both in the active and passive conditions, and parietal activity was present during the former condition only. While not resolving the relationship between the activity in auditory and parietal cortex (which would require MEG recordings to be carried out both in the active and passive conditions), our findings indicate that localization accuracy may already be determined in the auditory areas of the temporal lobe. Tentatively, the role of parietal areas could be to utilize the spatial information from the auditory areas to produce the appropriate motor response-without necessarily contributing to localization accuracy per se.

To summarize, the auditory cortices in both hemispheres reflect the processing of sound source location, although the right hemisphere appears to be specifically sensitive to 
spatial cues. Further, the activity of the right hemisphere in terms of the amplitude organization of the N1m correlates with behavioral localization accuracy, thus revealing a link between behavioral performance and the activity in auditory cortical brain areas. Finally, the source location of this brain activity reflects whether auditory stimuli are perceived as originating inside or outside the head.

\section{Acknowledgment}

This study was supported by the Academy of Finland (proj. no. 1201602, 180957, and 1277811).

\section{References}

[1] D.R. Begault, 3-D Sound for Virtual Reality and Multimedia, Academic Press Inc., Boston, 1994.

[2] J. Blauert, Spatial Hearing, revised ed., The MIT Press, Cambridge, 1997.

[3] J.F. Brugge, R.A. Reale, J.E. Hind, The structure of spatial receptive fields of neurons in primary auditory cortex of cat, J. Neurosci. 16 (1996) 4420-4437.

[4] K.A. Burke, A. Letsos, R.A. Butler, Asymmetric performances in binaural localization of sound in space, Neuropsychologia 32 (1994) $1409-1417$.

[5] K.O. Bushara, R.A. Weeks, K. Ishii, M.-J. Catalan, B. Tian, J.P. Rauschecker, M. Hallet, Modality-specific frontal and parietal areas for auditory and visual spatial localization in humans, Nat. Neurosci. 2 (1999) 759-766.

[6] R.A. Butler, Asymmetric performances in monaural localization of sound in space, Neuropsychologia 32 (1994) 221-229.

[7] Y.E. Cohen, E.I. Knudsen, Maps versus clusters: different representations of auditory space in the midbrain and forebrain, Trends Neurosci. 22 (1999) 128-135.

[8] L.Y. Deouell, N. Soroker, What is extinguished in auditory extinction? NeuroReport 11 (2000) 3059-3062.

[9] L.Y. Deouell, S. Bentin, N. Soroker, Electrophysiological evidence for an early (pre-attentive) information processing deficit in patients with right hemisphere damage and unilateral neglect, Brain 123 (2000) $353-365$.

[10] J.J. Eggermont, C.W. Ponton, The neurophysiology of auditory perception: from single units to evoked potentials, Audiol. NeuroOtol. 7 (2002) 71-99.

[11] N. Fujiki, K.A.J. Riederer, V. Jousmäki, J.P. Mäkelä, R. Hari, Human cortical representation of virtual auditory space: differences between sound azimuth and elevation, Eur. J. Neurosci. 16 (2002) 2207-2213.

[12] D.W. Grantham, Spatial hearing and related phenomena, in: B.J.C. Moore (Ed.), Hearing, Handbook of Perception and Cognition, 2nd ed., Academic Press, London, 1995, pp. 297-345.

[13] T.D. Griffits, G.G.R. Green, Cortical activation during perception of a rotating wide-field acoustic stimulus, NeuroImage 10 (1999) 84-90.

[14] H.E. Heffner, The role of macaque auditory cortex in sound localization, Acta Oto-Laryngol., Suppl. 532 (1997) 22-27.

[15] M. Hämäläinen, R. Hari, R.J. Ilmoniemi, J. Knuutila, O.V. Lounasmaa, Magnetoencephalography - theory, instrumentation, and applications to noninvasive studies of the working human brain, Rev. Mod. Phys. 65 (1993) 413-497.

[16] IEC-268-13: Sound System Equipment. Part 13: Listening Tests on Loudspeakers, 1985

[17] K. Itoh, M. Yumoto, A. Uno, T. Kurauchi, K. Kaga, Temporal stream of cortical representation for auditory spatial localization in human hemispheres, Neurosci. Lett. 292 (2000) 215-219.
[18] W.M. Jenkins, R.B. Masterton, Sound localization: effects of unilateral lesions in central auditory system, J. Neurophysiol. 47 (1982) 987-1016.

[19] I.P. Jääskeläinen, J. Ahveninen, G. Bonmassar, A.M. Dale, R.J. Ilmoniemi, S. Levänen, F.-H. Lin, P. May, J. Melcher, S. Stufflebeam, H. Tiitinen, J.W. Belliveau, Human posterior auditory cortex gates novel sounds to consciousness, Proc. Natl. Acad. Sci. U. S. A. 101 (2004) 6809-6814.

[20] J. Kaiser, W. Lutzenberger, N. Birbaumer, Simultaneous bilateral mismatch response to right - but not leftward sound lateralization, NeuroReport 11 (2000) 2889-2892.

[21] J. Kaiser, W. Lutzenberger, H. Preissl, H. Ackermann, N. Birbaumer, Right-hemisphere dominance for the processing of sound-source lateralization, J. Neurosci. 20 (2000) 6631-6639.

[22] A.J. King, J.W.H. Schnupp, T.P. Doubell, The shape of ears to come: dynamic coding of auditory space, Trends Cogn. Sci. 5 (2001) 261-270.

[23] D.J. Kistler, F.L. Wightman, A model of head-related transfer functions based on principal components analysis and minimumphase reconstruction, J. Acoust. Soc. Am. 91 (1992) 1637-1647.

[24] S. Kuwada, T.C.T. Yin, Physiological studies of directional hearing, in: W.A. Yost, G. Gourevitch (Eds.), Directional Hearing, SpringerVerlag, New York, 1987, pp. 146-176.

[25] S. Martinkauppi, P. Rämä, H.J. Aronen, A. Korvenoja, S. Carlson, Working memory of auditory localization, Cereb. Cortex 10 (2001) 889-898.

[26] P.J.C. May, H. Tiitinen, The MMN is a derivative of the auditory N100 response, Proceedings of the 14th International Conference on Biomagnetism (Biomag 2004), Aug. 8-12, 2004, Boston, USA, 2004.

[27] P. May, H. Tiitinen, R.J. Ilmoniemi, G. Nyman, J.G. Taylor, R. Näätänen, Frequency change detection in human auditory cortex, J. Comput. Neurosci. 6 (1999) 99-120.

[28] L. McEvoy, R. Hari, T. Imada, M. Sams, Human auditory cortical mechanisms of sound lateralization: II. Interaural time differences at sound onset, Hear. Res. 67 (1993) 98-109.

[29] J.C. Middlebrooks, J.M. Zook, Intrinsic organization of the cat's medial geniculate body identified by projections to binaural responsespecific bands in the primary auditory cortex, J. Neurosci. 3 (1983) 203-224.

[30] B.C.J. Moore, B.R. Glasberg, A revision of Zwicker's loudness model, Acta Acust. 82 (1996) 335-345.

[31] R. Näätänen, T. Picton, The N1 wave of the human electric and magnetic response to sound: a review and an analysis of the component structure, Psychophysiology 24 (1987) 375-425.

[32] K. Palomäki, P. Alku, V. Mäkinen, P. May, H. Tiitinen, Sound localization in the human brain: neuromagnetic observations, NeuroReport 11 (2000) 1535-1538.

[33] K. Palomäki, H. Tiitinen, V. Mäkinen, P. May, P. Alku, Cortical processing of speech sounds and their analogues in a spatial auditory environment, Cogn. Brain Res. 14 (2002) 294-299.

[34] C. Pantev, B. Lütkenhoner, M. Hoke, K. Lehnertz, Comparison between simultaneously recorded auditory-evoked magnetic fields and potentials elicited by ipsilateral, contralateral and binaural tone burst stimulation, Audiology 25 (1986) 54-61.

[35] C. Pantev, B. Ross, P. Berg, T. Elbert, B. Rockstroh, Study of the human auditory cortices using a whole-head magnetometer: left vs. right hemisphere and ipsilateral vs. contralateral stimulationl, Audiol. Neuro-Otol. 3 (1998) 183-190.

[36] R.D. Patterson, The sound of a sinusoid: spectral models, J. Acoust. Soc. Am. 96 (1994) 1409-1418.

[37] M. Reite, J.T. Zimmerman, J.E. Zimmerman, Magnetic auditory evoked fields: interhemispheric asymmetry, Electroencephalogr. Clin. Neurophysiol. 51 (1981) 388-392.

[38] M. Reite, M. Adams, J. Simon, P. Teale, J. Sheeder, D. Richardson, R. Grabble, Auditory M100 component 1: relationship to Heschl's gyri, Congn. Brain Res. 2 (1994) 13-20.

[39] F.K. Samson, P. Barone, A. Irons, J.C. Clarey, P. Poirier, T.J. Imig, 
Directionality derived from differential sensitivity to monaural and binaural cues in the cat's medial geniculate body, J. Neurophysiol. 84 (2000) 1330-1345.

[40] E. Schröger, Interaural time and level differences: integrated or separated processing? Hear. Res. 96 (1996) 191-198.

[41] T.M. Shackleton, R. Meddis, M.J. Hewitt, Across frequency integration in a model of lateralization, J. Acoust. Soc. Am. 91 (1992) 2276-2279.

[42] L. Stenbacka, S. Vanni, K. Uutela, R. Hari, Comparison of minimum current estimate and dipole modeling in the analysis of simulated activity in the human visual cortices, NeuroImage 16 (2002) 936-943.

[43] S.S. Stevens, E.B. Newman, Localization of actual sources of sound, Am. J. Psychol. 48 (1936) 297-306.

[44] Y. Takashi, M. Konishi, Manipulation of inhibition in the owl's nucleus laminaris and its effects on optic tectum neurons, Neuroscience 111 (2002) 373-378.

[45] W. Teder-Sälejärvi, S. Hillyard, The gradient of spatial auditory attention in free field: an event-related potential study, Percept. Psychophys. 60 (1998) 1228-1242.

[46] P. Ungan, S. Yagiciogly, C. Goksoy, Differences between N1 waves of the responses to interaural time and intensity disparities: scalp topography and dipole sources, Clin. Neurophysiol. 112 (2001) 485-498.
[47] K. Uutela, M. Hämäläinen, E. Somersalo, Visualization of magnetoencephalographic data using minimum current estimates, NeuroImage 10 (1999) $173-180$.

[48] R.A. Weeks, A. Aziz-Sultan, K.O. Bushara, B. Tian, C.M. Wessinger, N. Dang, J.P. Rauschecker, M. Hallet, A PET study of human auditory spatial processing, Neurosci. Lett. 262 (1999) 155-158.

[49] E.M. Wenzel, M. Arruda, D.J. Kistler, F.L. Wightman, Localization using nonindividualized head-related transfer functions, J. Acoust. Soc. Am. 94 (1993) 111-123.

[50] F.L. Wightman, D.J. Kistler, Headphone simulation of free-field listening: I. stimulus synthesis: II. psychophysical validation, J. Acoust. Soc. Am. 85 (1989) 858-878.

[51] M.G. Woldorff, C. Tempelmann, J. Fell, C. Tegeler, B. GaschlerMarkefski, H. Hinrichs, H.J. Heinze, H. Scheich, Lateralized auditory spatial perception and the contralaterality of cortical processing as studied with functional magnetic resonance imaging and magnetoenchephalography, Hum. Brain Mapp. 7 (1999) 49-66.

[52] J.R. Wolpaw, J.K. Penry, Hemispheric differences in the auditory evoked response, Electroencephalogr. Clin. Neurophysiol. 43 (1977) 99-102.

[53] R.J. Zatorre, V.B. Penhune, Spatial localization after excision of human auditory cortex, J. Neurosci. 21 (2001) 6321-6328.

[54] R.J. Zatorre, M. Bouffard, P. Ahad, P. Belin, Where is 'where' in the human auditory cortex? Nat. Neurosci. 5 (2002) 905-909. 